\title{
MontePython 3: boosted MCMC sampler and other features
}

Thejs Brinckmann \& Julien Lesgourgues

Institute for Theoretical Particle Physics and Cosmology (TTK), RWTH Aachen University, Otto-Blumenthal-Strasse, 52057, Aachen, Germany. E-mail: brinckmann@physik.rwth-aachen.de:lesgourg@physik.rwth-aachen.de

\begin{abstract}
MontePython is a parameter inference package for cosmology. We present the latest development of the code over the past couple of years. We explain, in particular, two new ingredients both contributing to improve the performance of Metropolis-Hastings sampling: an adaptation algorithm for the jumping factor, and a calculation of the inverse Fisher matrix, which can be used as a proposal density. We present several examples to show that these features speed up convergence and can save many hundreds of CPU-hours in the case of difficult runs, with a poor prior knowledge of the covariance matrix. We also summarise all the functionalities of MontePython in the current release, including new likelihoods and plotting options.
\end{abstract}

ก

Keywords: Cosmology: -parameter inference, -numerical tools 


\section{Introduction}

MontePython [1] is an MCMC sampling package in Python used for parameter inference in cosmology, similar to CosmomC [2, 3] and CosmoSIS [4]. The modular nature of MontePython means modification of the code is particularly easy, and encourages implementation of specific modules to other Python sampling packages, e.g. the extensive library of cosmological likelihoods 1 . MontePython has two different modes: when running with

$>$ python montepython/MontePython run <options>

it is a sampler (similar to CosmoMC), and when running with

$>$ python montepython/MontePython info <options>

it is a tool for analyzing MCMC chains and plotting results (similar to GetDist ${ }^{2}$ ).

The code is currently interfaced with the Boltzmann code CLASS [5-8] and extensions thereof, e.g. HiCLASS [9] and SONG [10]. There also exist some publicly available branches of CLASS achieving different purposes, e.g. ExoCLASS for advanced energy injection, recombination and reionisation features [11], CLASS_SZ for SunyaevZel'dovich observables [12], CLASSgal for computing the number count $C_{\ell}$ 's [13] $]^{3}$, or a branch incorporating nonlocal contributions to General Relativity [14 $]^{4}$.

In principle, it could easily be extended for use with e.g. CAMB [15, 16], via the new Python wrapper ${ }^{5}$, or PyCosmo [17], a Boltzmann code in Python.

In this paper, we present the latest development of MontePython over the past couple of years. In particular, we introduce two new ingredients that both contribute towards improving the performance of Metropolis-Hastings sampling. In Section 2, after recalling the way in which the Metropolis-Hastings algorithm is implemented in MontePython, we present a new adaptation algorithm for the jumping factor. In Section 3 , we detail our strategy for calculating the Fisher matrix and its inverse, which can be used as a proposal density for a Metropolis-Hastings run. In Section 4, we provide several examples of runs showing that these features speed up convergence and can save many hundreds of CPU-hours in the case of difficult runs, with a poor prior knowledge of the covariance matrix. In the various appendices, we summarise all the functionalities of MontePython in the current release, including extended cosmological parameter definitions with respect to CLASS in Appendix A, sampling options in Appendix B, analysis and plotting options in Appendix C and likelihoods in Appendix D Indeed, the new release of the code incorporates several new plotting options and even more new likelihoods based either on current or mock data.

The version of the code described in this paper has version number 3.0 and is available at https://github.com/ brinckmann/montepython_public

\section{Metropolis-Hastings sampling strategy}

MontePython can switch between different ways to explore parameter space, which include Metropolis-Hastings, Nested Sampling, Cosmo Hammer, and a new Fisher sampling method described in section 3 . These different algorithms are called methods in the code, and the same list of methods also includes post-processing algorithms like Importance Sampling or Adding Derived Parameter(s)

The default method is the Metropolis-Hastings algorithm, working since v2.0.0 (2013) with a fast sampling method quickly summarised in section 2.1, and since v2.2.0 (October 2015) with a covariance matrix update method summarised in section 2.4. In this release v3.0.0 we extend the latter to update also the jumping factor, as described in section 2.4 and we call the new approach superupdate.

\footnotetext{
${ }^{1}$ A practice the authors fully support and encourage, with proper citations and credits.

${ }^{2}$ http://getdist.readthedocs.io/en/latest/

${ }^{3}$ this feature has also be implemented later in the main CLASS, but with small differences in the two implementations. The original CLASSgal code is still available at https://cosmology.unige.ch/content/classgal

${ }^{4}$ see the pull request \#86 in https://github.com/lesgourg/class_public

5 http://camb.readthedocs.io/en/latest/

${ }^{6}$ The full list of methods can be viewed with MontePython. py --run --help, and is of the form -m \{MH, NS, CH, IS, Der, Fisher\}.
} 


\subsection{Fast sampling}

In MontePython the Metropolis-Hastings draws random jumps in parameter space from a Gaussian proposal density. The latter is encoded in a matrix $\mathbf{C}$, describing the parameter correlations and the standard deviations relative to each other, and an overall jumping parameter $c$, such that the parameter jumps $\Delta \mathbf{p}$ are generated randomly from the probability distribution $\mathcal{P}=\mathcal{N} \exp \left(-\frac{1}{2 c} \Delta \mathbf{p}^{T} \mathbf{C}^{-1} \Delta \mathbf{p}\right)$. Thus the actual covariance matrix of the proposal density is $c \mathbf{C}$. The standard way to generate random vectors from a multivariate Gaussian probability is to go to a basis of independent parameters, like the basis of the eigenvectors of $\mathbf{C}$; to generate independent random displacements along the eigenvectors; and to project back to the original space.

It is well-known that optimal proposal densities generate an acceptance rate of the order of 0.25 , and that for Gaussian posterior distributions, this can be achieved when $\mathbf{C}$ is a good approximation to the covariance matrix of the posterior distribution, while the jumping parameter is fixed to $(2.4)^{2}$ [18]. Note that this jumping parameter applies when generating one single random number and moving in one single direction. Alternatively, for each jump, one can generate $N$ random numbers and move in $N$ directions simultaneously, but then each of these $N$ random numbers should be drawn from a Gaussian distribution with variance $c=j^{2} / N$, where $j=2.4$ is called the jumping factor.

Thus, in absence of fast sampling, the jumps could just be generated each time independently and randomly from $\mathcal{P}$ (global method), or in cycles of $N$ draws along each of the $N$ eigenvectors of $\mathbf{C}$ (sequential method). These methods can still be activated 7 in MontePython, but they are sub-optimal in presence of likelihoods with nuisance parameters.

Fast sampling was proposed by [3] for MCMC parameter estimation. For this sampling method, we separate the sampling of fast nuisance and slow cosmological parameters to optimize performance when dealing with a large number $N_{\text {fast }}$ of nuisance parameters.

Generating displacements along the eigenvectors mixes slow and fast parameters and does not allow for high-speed explorations of the fast parameter space only. But introducing eigenvectors is not the only way to go to a parameter basis in which the proposal density is orthogonal. In particular, one can perform a Cholesky decomposition of the covariance matrix into $\mathbf{C}=\mathbf{L} \mathbf{L}^{T}$ where $\mathbf{L}$ is a lower triangular matrix. In the space of the vectors $\Delta \mathbf{p}^{\prime}$ related to the physical parameters through $\Delta \mathbf{p}^{\prime}=\mathbf{L}^{-1} \Delta \mathbf{p}$, the proposal density is orthogonal, so the jumps can easily be generated by drawing random numbers for each component of $\Delta \mathbf{p}^{\prime}$ with a single one-dimensional Gaussian probability distribution of variance $c$, and projecting back to $\Delta \mathbf{p}=\mathbf{L} \Delta \mathbf{p}^{\prime}$. The great advantage over the previous eigenvector-based scheme is that when $\Delta \mathbf{p}^{\prime}$ only has non-zero components above a given index, this is true also for $\Delta \mathbf{p}$. Thus one can generate some jumps that will leave the slow parameters unchanged.

We begin by ordering our input parameters in blocks according to computational time. In practise, this is simply achieved by writing them in the right order in the input parameter file. The first block is that of cosmological parameters requiring new calls to the Boltzmann code. The next blocks are nuisance parameters for a given likelihood, which can be changed without requiring a Boltzmann code evaluation if the cosmological parameters are held fixed. The nuisance parameter blocks should be ordered from the slowest to fastest likelihood. When a nuisance parameters is common to several likelihoods, it should just be declared within the slowest block. We call $M$ the number of blocks and $d_{j}$ the number of parameters in the $j$-th block, with $d_{1}=N_{\text {slow }}$ being the number of cosmological parameters. MontePython will automatically detect the number $M$ of blocks and will expect the user to pass an $M$-dimensional over-sampling vector $\mathbf{F}$. $F_{1}$ is the over-sampling factor of the cosmological parameter and is normally fixed to one. The other entries are the required number of redundent sampling for each of the other blocks.

When running chains, for $j=1, \ldots, M$, we generate sequences of $F_{j} d_{j}$ random jumps in the $d_{j}$ components of $\Delta \mathbf{p}^{\prime}$ corresponding to the $j$-th block. In other words, during $F_{j} d_{j}$ steps, we generate $d_{j}$ random numbers for each of the relevant components of $\Delta \mathbf{p}^{\prime}$, drawn from a Gaussian distribution with standard variance $c=j^{2} / d_{j}$. Thus each full cycle consists of $\sum_{j} F_{j} d_{j}$ random jumps, with only $F_{1} d_{1}=N_{s}$ of them requiring a call to the Boltzmann code. Later we will call this number the Fast Parameter Multiplier (FPM):

$$
\mathrm{FPM}=\sum_{j} F_{j} d_{j}
$$

There is no precise rule to fix the over-sampling factors $F_{2, \ldots, N}$. These factors should be increased for faster likelihoods and/or larger numbers of nuisance parameters in the block. With too low numbers, one would not enjoy the advantages

${ }^{7}$ with the jumping flag $-j$ \{global, sequential\} instead of the default $-j$ fast. 
of the slow-fast parameter decomposition. With too high numbers, the time spent in the $\left(\sum_{j} F_{j} d_{j}-N_{s}\right)$ iterations over fast parameters could be significant compared to the time spent in the $N_{s}$ iterations over slow parameters, and the convergence of the results for the cosmological parameter would be delayed. For instance, for the nuisance parameters of the Planck likelihood, we usually apply an oversampling factor of 4 .

\subsection{Update and Superupdate}

While the Metropolis-Hastings algorithm would in principle require Markov Chains, i.e. chains with a proposal density that is constant in time, it is highly desirable to implement some automatic update algorithms in order to get converged results even when starting from bad guesses for the Gaussian proposal density. We recall that the propoosal density is parametrised as $\mathcal{P}=\mathcal{N} \exp \left(-\frac{1}{2 c} \Delta \mathbf{p}^{T} \mathbf{C}^{-1} \Delta \mathbf{p}\right)$ and thus depends on two quantitites, the covariance matrix $\mathbf{C}$ and the jumping parameter $c$ related to the jumping factor $j$. MontePython has two complementary options for speeding up the convergence of Metropolis-Hastings runs:

- --update $U$ : update of covariance matrix, $\mathbf{C}$, every $U$ cycles [default: $U=50$ ]

- --superupdate $S U$ : additionally, update of jumping factor, $j$, starting $S U$ cycles after each covariance matrix update [default: $S U=0$, meaning "deactivated"; recommended: 20]

Once certain criteria are met, the covariance matrix will be updated periodically and the jumping factor will be adapted every step. This leads to dramatic improvements in runtime, especially for runs with little prior knowledge in the form of an appropriate starting stepsize or a good initial proposal distribution. In the next two sections we describe the strategy chosen for these two schemes.

\subsection{Update strategy}

The covariance matrix update mechanism was implemented early on in CosmoMC [], and is part of MontePython since v2.2.0 (October 2015), through the flag --update. The MontePython and CosmoMC implementations of this feature are very similar. For instance, in both codes, the decision to start or stop the update mechanism depends on the value of the Gelman-Rubin statistic, $R$ [19], for the most poorly converged parameter. The update starts when the number $\max (R-1)$ computed from the second half of each chain goes below 3 , and stops when it goes below 0.4. The difference between the two implementations only resides in two aspects:

- Non-MPI-user friendliness. The most straightforward way to launch multiple chains is to run MontePython with MPI, e.g. for 8 chains: mpirun -np 8 python montepython/MontePython.py run ...

The alternative would be to launch 8 chains manually, or within a small shell script with a for loop. With CosmoMC, this second option would be incompatible with the covariance matrix updating. In MontePython, because installing MPI can sometimes be cumbersome, we chose to code the --update mechanism in such a way that it will work equally well with or without MPI. In the latter case, the user should just run several times python montepython/MontePython.py run ..., and the update mechanism will still start and use the information from all the chains running in the same directory

- Keeping only Markovian steps in the final results. To be rigorous, the user would like to base his final results and plots on true Markovian chains. This is what happens by default with MontePython. Indeed, every time that the covariance matrix is updated, the code writes in all chains files a comment line starting with \# After <k> accepted steps: update proposal...

\footnotetext{
${ }^{8}$ There is no need to know how this is implemented in the code, because it is fully transparent to users. In brief, the key point is that MontePython reads and writes the covariance matrix in a file, rather than using the Message-Passing-Interface. The only difference between MPI and non-MPI runs is that in the former case, the code can define a "master chain" and some "slave chains", and only the master chain occasionally pauses in order to update the covariance matrix; in the latter case, all chains occasionally pause for the same purpose, but this does not affect convergence, and it only increases the total running time by a very small amount. Note that exchanging information on the covariance matrix through a file could have a potential inconvenience: When the user analyses an on-going run with the info mode, she/he could generate a new covariance matrix that may interfere with the automatic updating mechanism. This is not the case because when the user runs in info mode, the covariance matrix calculation is de-activated by default; it is only activated with the --want-covmat flag.
} 
which also contains information on the current convergence (thus these comments can also be used to scrutinise what is happening with the run). When analysing the chains with the info mode, by default, the code will only consider the part of the chains after the last update, i.e. the Markovian part. If the user wants to de-activate this behaviour in order to get more points in the chains, she/he can use the flag--keep-non-markovian.

Finally, the update periodicity is controlled by the $U$ input parameter (default: $U=50$ ), which is in units of cycles. Given that a cycle consists in FPM steps (see eq. (1)), the update takes place every

$$
N_{\text {update }}=\mathrm{U} \times \mathrm{FPM}
$$

steps (here we are referring to proposed steps, not accepted steps).

\subsection{Superupdate strategy}

The covariance matrix updating does not fully achieve the task of reaching optimal convergence conditions automatically. The other part of the proposal density is the jumping parameter $c$, related to the jumping factor $j$. If $j$ is too large, the acceptance rate (a.r.) is too small and the number of accepted models remains insufficient to extract statistical information. If $j$ is too small, the a.r. may get close to one. In that situation the chains would grow rapidly, but adjacent points would be very correlated, and the chains would not necessarily sample the full posterior distribution. Thus one should target a compromise value of the acceptance rate. Since the work of [18], cosmologists usually aim at $a . r . \simeq 0.25$ (although larger values in the range 0.3-0.5 would in principle still be acceptable).

While $f=2.4$ matches this goal for a multivariate Gaussian posterior, many runs target non-Gaussian posteriors, e.g. due to non-trivial priors on cosmological parameters (like the requirement of a positive neutrino mass) or to strongly non-Gaussian posteriors for the nuisance parameters (like those of the Planck high- $\ell$ likelihoods). The current practise consists in training a bit and trying manually different values of $f$ until the acceptance rate is correct. For instance, one quickly comes to know that, e.g., a given version of the Planck likelihood usually needs a given value of $f$ to achieve $a . r . \simeq 0.25$. Of course, it would be better to let the code find this value automatically for each combination of a model and a dataset. An automatic jumping factor adaptation would also make the code more powerful when starting from a very bad proposal density (e.g., when adding many new free parameters to a previous run, or when investigating a very constraining set of likelihoods when only the covariance matrix of a much less constraining set is available).

For that purpose, we added to version v3.0.0 the new option--superupdate, which is complementary to --update: they should normally be used in combination, but then it is only necessary to pass the --superupdate flag since--update is activated by default.

Note that other schemes to update the full proposal density (rather than just the covariance matrix) have been investigated in the past. For instance, an Adaptative Metropolis algorithm for single-chain runs was proposed in [20], and a version of this algorithm was implemented in a cosmology MCMC code by the CAMEL collaboration [21]. After trying this method for single chains, we adapted it freely to the multi-chain case, in a way which remains compatible with the traditional covariance matrix update scheme (--update).

Overall strategy. When running with --superupdate $S U$, the code starts from a jumping factor that can be set manually with a command flag (e.g. --f 2.2), but when nothing is passed, 2.4 is used by default. The code keeps a record of the acceptance rate, a.r., and of the jumping parameter, $c$, of the last $S U$ cycles, i.e. of the last $S U \times$ FPM steps. It also keeps track of the average $\overline{a . r}$. and $\bar{c}$ over these last $S U$ cycles. This information is used to compute the start- and stop-criteria. Since--superupdate requires --update to be active, the run can be divided in several "update sequences", which are the ensemble of steps between two consecutive updates. The first "update sequence" is just the time until the first update. The basic principle of superupdate is to adapt the jumping factor at each step according to the recurrence relation

$$
c_{k}=c_{k-1}+\frac{1}{\left(k-k_{\text {update }}\right)}(\overline{a . r .}-0.26)
$$

where $k$ is the current step number, while $k_{\text {update }}$ is the first step number of each new "update sequence". For the first sequence, $k_{\text {update }}=0$. This recurrence relation leads to faster updating at the beginning of each new sequence, and to 
slower updating and safe convergence properties after some time.

Starting the jumping factor update. The code starts applying the recursion relation (3) when two conditions are met:

- We do not want to update the proposal distribution too early, as it could be based on chains still in the burn-in phase. For this reason, we wait until the chains have reached a certain level of convergence: the numbers $(R-1)$ computed from the second half of each chain should be below 10 for all parameters.

- We wait until we have done $S U$ cycles since the beginning of the new "update sequence", or since the very beginning if we are still in the first sequence: $\left(k-k_{\text {update }}\right) \geq S U \times F P M$. Since the mean acceptance rate $\overline{a . r}$. is computed over the last $S U$ cycles, the recurrence will only take into account some steps from the same "update sequence". Choosing $S U \gtrsim 20$ ensures that the mean acceptance rate is not computed over too small a sample, where shot noise may lead to an acceptance rate significantly different from the target one (i.e. due to random fluctuations in the acceptance rate leading to prematurely stopping adaptation of the jumping factor). We recommend using $S U=20$, as we found this to be a good compromise between efficiency and precision, but higher values can be considered in order to decrease the impact of superupdate (the jumping factor would start evolving later and would perform smaller excursions) or to further decrease the impact of shot noise on the determination of the jumping factor. Note that with $S U>U$, superupdate would sometimes only be active after the very last update of the covariance matrix, in the final stage of the run, when the convergence is already good (or possibly before the chains are well enough converged for updating the covariance matrix to begin). Thus one should normally consider the range $20 \leq S U<U$ only.

Rescaling when the covariance matrix gets updated. Since the true covariance matrix of the Gaussian proposal density is in fact given by the product $c \mathbf{C}$, it would be sub-optimal to leave $c$ unchanged when the matrix $\mathbf{C}$ is updated at the beginning of each new "update cycle". Suppose for instance that in the $n$-th "update cycle", a good jumping factor $c_{n}$ has been found in combination with a covariance matrix $\mathbf{C}_{n}$ (by this we mean that the acceptance rate has the correct order of magnitude). If at the beginning of the next cycle the matrix is adapted to a $\mathbf{C}_{n+1}$ which is much smaller, while $c_{n+1}$ restarts from the same value $c_{n}$, then obviously the whole proposal density will shrink and the acceptance rate will increase too much. We can limit this effect by requiring analytically that at each covariance matrix update, the volume probed by the full proposal density remains constant, which is achieved simply by imposing:

$$
c_{\text {after }}^{N} \operatorname{det}\left(C_{\text {after }}\right)=c_{\text {before }}^{N} \operatorname{det}\left(C_{\text {before }}\right),
$$

where $N$ is the number of free (slow+fast) parameters. This rescaling might not be very efficient when the evolution of the covariance matrix comes from only one or few parameters, but in general, it is the best simple guess that one can do. In terms of the jumping factor, this gives:

$$
j_{\text {after }}=j_{\text {before }}\left[\frac{\operatorname{det}\left(C_{\text {before }}\right)}{\operatorname{det}\left(C_{\text {after }}\right)}\right]^{\frac{1}{2 N}} .
$$

Note that, for the first update of the covariance matrix, the logic behind this re-scaling does not hold. Indeed, if we started from a poor input covariance matrix, the first re-scaling of the jumping factor may be completely unrealistic. For safety, at the first update time, we reset the jumping factor to the input value (provided via --f [default: 2.4$]$ ).

Stopping the jumping factor update. We adapt the jumping parameter until three conditions are met:

- The a.r. should converge to $26 \%$ with a tolerance of 1 percent poin 9 (in many cases, the a.r. starts low and increases to the optimal value, then the adaptation will stop when the code reaches $25 \%$ ):

$$
|\overline{a . r .}-0.26|<0.01
$$

\footnotetext{
${ }^{9}$ This behaviour is controllable by the options --superupdate-ar and --superupdate-ar-tol.
} 
- In addition to the a.r. criterium, in order to stop adaptation of the jumping parameter we also require that it is stable,

$$
\left|\frac{\bar{c}}{c_{k-1}}-1\right|<0.01
$$

where $\bar{c}$ is the mean of the jumping parameter over the last $S U \times$ FPM steps. Also, we do not wish to allow the jumping parameter to converge to arbitrarily low values, as the risk of chains getting stuck in local minima would increase. Therefore, we introduce a minimum for the jumping factor corresponding to $10 \%$ of the initial jumping factor. If a small jumping parameter is desired, it is instead recommended to input a low value with $--f$.

- Finally, we require that the number $\max (R-1)$ computed from the second half of the chains is below 0.4 for all parameters: this is the same condition as for stopping the covariance matrix update. Thus the superupdate mechanism will only stop its activity in the final "update sequence", during which a large number of truly Markovian steps (generated with a constant proposal density) can be accumulated.

Non-MPI user friendliness. We implemented the superupdate mechanism in MontePython with the same coding principles as for the update mechanism. Thus it can also be used with or without MPI, thanks to the fact that the communication between chains works through files rather than MPI commands. In the running directory, a file jumping_factors.txt stores the sequence of all jumping factors that have been used, while the file jumping_factor.txt only contains the final one, that can be used as an input value in the next run. When chains are restarted in the same directory using the --restart command, this will be done automatically.

Keeping only Markovian steps in the final results. When --superupdate is activated, the code still writes some comment lines in the chains at the beginning of each new "update cycle", with information on the current value of $\max (R-1), j$ and a.r.. Additionally, it writes an extra line of comments when the jumping factor updating stops. When analyzing the chains in info mode, and unless the user passes the option--keep-non-markovian, all the lines before will be discarded and the final numbers and plots will be based on purely Markovian chains.

Alternative implementation for single chain runs. The superupdate mechanism in principle requires multiple chains, since it uses convergence tests based on the Gelman-Rubin statistic. For single chain runs the code will split the chain into three separate chains in order to compute the Gelman-Rubin statistic, a practice that may be less reliable than running multiple chains. However, MontePython also has an alternative to superupdate that was previously implemented for single chain runs by [22]. This other mechanism is activated by the flag--adaptive instead of --superupdate. It does not use the Gelman-Rubin statistic, and it is slightly closer to the original Adaptive Metropolis algorithm of [20], with an update of the covariance matrix at each single step.

\section{Fisher matrix}

The well-known Fisher matrix is built from the second derivatives of the effective $\chi^{2}$ with respect to the model parameters computed at a minimum of the $\chi^{2}$, i.e. at the maximum of the likelihood (see e.g. [23]):

$$
\mathbf{F}_{i j}=\frac{1}{2} \frac{\partial^{2} \chi^{2}}{\partial p_{i} \partial p_{j}}=-\frac{\partial^{2} \ln \mathcal{L}}{\partial p_{i} \partial p_{j}} .
$$

By definition of the maximum likelihood point, the Fisher matrix must be positive definite and invertible. Its inverse is the covariance matrix of the Gaussian approximation to the likelihood near the best-fit point. If the matrix of second derivatives is not computed at that point, it may not be invertible.

\subsection{Motivations for Fisher matrix computation}

We implemented in MontePython v3.0.0 a calculation of the Fisher matrix directly from the likelihood and from eq. (8), using a finite difference method that we will detail in the next section. The motivation behind this calculation is twofold: 
Boosting MCMC runs. The inverse Fisher matrix can be used as the input covariance matrix of an MCMC run (e.g. Metropolis-Hastings). In that case we don't need a high-accuracy calculation of this matrix, because any approximate result will likely be a good enough guess, that the Metropolis-Hastings "update" mechanism will quickly improve anyway. This method leads to a very significant speed up in most cases, since one rarely starts an MCMC run with already at disposal a very good covariance matrix including all pairs of parameters. Still, the method can work only if the code finds an invertible Fisher matrix in the first place, and this is only guaranteed at the exact maximum likelihood point. This condition can be easy or difficult to achieve depending on the type of run:

- For parameter forecasts with mock data, we usually use the fiducial spectra in the role of the observed spectra, without generating a random realisation (see e.g. [24] for comments on this methodology). Thus the maximum likelihood exactly coincides with the fiducial model, known in advance by the user. Then the new Fisher method works particularly well.

- For parameter extraction from real data, we know at most an approximation to the best fit point. Then, one may hope that if the distance between the true and approximate best fit points is small compared to the steps used in the finite difference method, the approximate Fisher matrix computed in the latter point will still be positive definite and invertible. We found however that this does not happen very often, so the possibility to use this new method for real data remains somewhat random. To increase chances, we incorporated in MontePython a few minimum-finding algorithms taken from the optimize python library ${ }^{10}$ that may at least help to get closer to the true best-fit point before trying the Fisher matrix computation. However, our tests show that, for the moment, the implemented minimization algorithms are not very robust, especially in the presence of many nuisance parameters.

In summary, the new Fisher calculation definitely improves all MCMC forecasts (as shown in section 4), and it may also improve MCMC runs with real data (see a few examples in section 4.2) unless one ends up with likelihood shapes that happen to be too complicated for finding the minimum and/or running the Fisher algorithm.

Replacing MCMC runs. When one knows that the posterior of a given run should be nearly Gaussian, or when one is not interested in the details of the posterior (e.g. non-trivial parameter correlations with some skewness, kurtosis, banana-shape, etc.), it is tempting to replace whole MCMC parameter extraction runs by simple Fisher matrix computations. Then the inverse Fisher matrix will give some approximate one-dimensional confidence regions and two-dimensional elliptic contours. This is particularly straighforward for sensitivity forecasts since, in that case, the maximum likelihood point is known in advance. It can also be envisaged for real data if the maximum likelihood point is known up to good approximation, for instance, after a run with the new --minimize option of MontePython (whose success is not guaranteed).

In the cosmology literature, a vast majority of parameter forecasts are based on Fisher matrix calculations. These are usually performed by specific codes, using the fact that after a few steps of analytic calculations, $F_{i j}$ can be reexpressed as a function of the derivative of the observable quantities with respect to the parameters (e.g. $\partial C_{\ell} / \partial p_{i}$ or $\left.\partial P(k) / \partial p_{i}\right)$. Instead, the Fisher matrix computation performed by MontePython is a direct likelihood-based evaluation, since we compute $\partial \ln \mathcal{L} / \partial p_{i}$. The two approaches are mathematically equivalent, but the latter may offer some practical avantages. Indeed, the quantity which is primarily build to model a given experiment is the likelihood. Skipping the analytical steps leading to derivatives like $\partial C_{\ell} / \partial p_{i}$ or $\partial P(k) / \partial p_{i}$ sometimes avoids complicated expressions, the need to introduce approximations, and further risks to make an error.

In both approaches, one has to compute some numerical derivatives with a given step size. For a purely Gaussian likelihood, the step size should be irrelevant, provided that it is not so small that numerical errors (from the Boltzmann code or from the likelihood code) come to dominate. Very often, Boltzmann codes are optimized in order to give an

\footnotetext{
${ }^{10}$ If the user runs MontePython with the command flag --minimize, before using any engine (Metropolis-Hastings, Fisher, etc.), the code will re-evaluate the central starting point using a $\chi^{2}$ minimization algorithm. This call is done in the routine get minimum() of the module montepython/sampler.py. After loading some approximation for the best-fit point and for the iteration step size, this routine calls the python function numpy .optimize.minimize(), which accepts several values of the input parameter method, corresponding to different minimization algorithms. By default we did set method='SLSQP', which calls the Sequential Least SQuares Programming algorithm, but the user is free to edit the module and change one line to try different methods. The algorithm stops when the $\chi^{2}$ seems to be converged up to the tolerance passed through the MontePython input flag --minimize-tol (default $10^{-5}$ ), but there is no guarantee that the algorithm leads to the true minimum.
} 
accuracy on the $\chi_{\text {eff }}^{2}$ of the most constraining experiments (typically, nowadays, the Planck experiment) of the order of $\delta \chi^{2} \sim \mathcal{O}\left(10^{-1}\right)$, simply because achieving better precision would not change the results on confidence intervals, and would thus be a waste of computing time. Therefore, it is dangerous to use steps $\Delta p_{i}$ such that

$$
\Delta \chi^{2} \equiv \frac{1}{2}\left(\ln \mathcal{L}\left(p_{i}^{\text {bestfit }}+\Delta p_{i}\right)-\mathcal{L}\left(p_{i}^{\text {bestfit }}\right)\right)
$$

is significantly smaller than 0.1 . This provides roughly a lower bound on the $\Delta p_{i}$ 's. The question of the upper bound is more delicate, and especially important when the likelihood is not Gaussian: different choices can then return significantly different Fisher matrices and confidence limits. The community is split between different approaches on this issue. One school suggests to take the smallest possible steps until numerical noise comes into play, in order to be as close as possible to the mathematical definition of the second derivatives. Another school prefers to choose steps such that $\Delta \chi^{2} \simeq 1$ (resp. 4) if the final goal is to deliver predictions for $68 \%$ (resp. 95\%) confidence limits on the parameters, since in that case the Fisher matrix gives a Gaussian approximation of the likelihood valid precisely in the region that is relevant for the parameter bounds (see e.g. [24]). Many Fisher codes do not even target any particular given order of magnitude for $\Delta \chi^{2}$, and choose the steps $\Delta p_{i}$ arbitrarily.

In MontePython v3.0.0, we approach this problem by letting the user choose a target value for $\Delta \ln \mathcal{L}=2 \Delta \chi^{2}$. By default, the code will first try to get an invertible Fisher matrix with $\Delta \ln \mathcal{L} \sim 0.1$, and will iteratively increase this value in case the result is non-invertible, as explained in the next section. However, the user can choose the first value of $\Delta \ln \mathcal{L}$, and may for instance set it to 0.5 or 2 (with the flag--fisher-delta).

\subsection{Iterative strategy for the fisher matrix computation}

The Fisher matrix calculation is a "method", like e.g. Metropolis-Hastings, Nested sampling, Importance Sampling, minimization, etc. It is activated by launching MontePython v3.0.0 in run mode with the command flag:

- --method Fisher : calculate Fisher matrix

The calculation takes place around parameter values specified by the first entries of each list in the input file:

$$
\text { data. parameters }\left[{ }^{\prime} \mathrm{P} 1{ }^{\prime}\right]=[\mathrm{p} 1, \ldots, \ldots, \ldots, \ldots, \ldots] \text {, }
$$

unless another best-fit model is passed with the command line -b path/to/file.bestfit. The user may control the step size for the finite difference derivatives with two parameters:

- --fisher-delta $D$ : target $\Delta \ln \mathcal{L}$ value for finding the steps $\Delta p_{i}$ [default: $D=0.1$ ]

- --fisher-tol $T$ : tolerance for $\Delta \ln \mathcal{L}$ (note: decreasing slows down computation) [default: $T=0.05$ ]

Then the code finds the step size for each parameter matching the target $\Delta \chi^{2}=D \pm T$ by bisection. Sometimes the bisection struggles to converge (e.g. for non-Gaussian likelihoods, or if the calculation is not centered on the maximum of the likelihood). In this case, after 10 attempts, it gradually increases the tolerance $T$ at each step until convergence is obtained. However, in such an event, it may be preferable to adjust the input parameters instead (e.g. target $D$ or best fit parameter values).

Once the step sizes have been obtained, the code computes all the elements of the Fisher matrix. If the result is a non-invertible matrix (due to the non-Gaussianity of the likelihood or to a bad guess for the maximum likelihood), the code enters into a stage of iterations over the target value of $\Delta \chi^{2}$, which is steadily increased until the matrix becomes invertible, following the sequence $D, 2 D, 3 D, \ldots N D$. The maximum number of iterations can be controlled with

- --fisher-step-it $N$ : number of step iterations attempted [default: $N=10$ ]

If the matrix inversion still fails after the maximum number of iterations, the code stops and returns an explicit error message.

Whenever the code finds an invertible Fisher matrix, it stores both the Fisher matrix and its inverse in distinct files with the extension .mat. The inverse Fisher matrix file matches the usual format of any covariance matrix that the Metropolis-Hastings algorithm would take in input for the density proposal. Thus it can immediately be used in an MCMC run with the input flag -c path/to/file.covmat. 


\subsection{Dealing with prior boundaries}

In the last section we mentioned that the code finds the step sizes $\Delta p_{i}$ used in numerical derivatives with a bisection algorithm. The bisection starts with a first tentative step size given by the input $\sigma_{i}^{\text {input }}$ value for a each parameter, as given by the input file or by the input covariance matrix specified by the input flag -c path/to/file. covmat (the second always has priority). In cases where a $p_{i}^{\text {bestfit }} \pm \sigma_{i}^{\text {input }}$ value exceeds the prior boundary, we change the initial step $\sigma_{i}^{\text {initial }}$ according to the following criteria:

Case 0: When there are no boundaries, or the difference between the boundary and the center is greater than $\sigma_{i}^{\text {input }}$, the initial step is given by $\sigma_{i}^{\text {input }}$ :

$$
\sigma_{i}^{\text {input }}<B_{i-} \text { and } \sigma_{i}^{\text {input }}<B_{i+} \Rightarrow \sigma_{i}^{\text {initial }}=\sigma_{i}^{\text {input }},
$$

where the lower boundary distance is $B_{i-}=p_{i}^{\text {bestfit }}-p_{i}^{\text {lower-boundary }}$ and the upper one is $B_{i+}=p_{i}^{\text {upper-boundary }}-p_{i}^{\text {bestfit }}$.

Case 1: When one or both of the boundary distances is smaller than $\sigma_{i}^{\text {input }}$, but both are still larger than a tenth of $\sigma_{i}^{\text {input }}$, we set the initial step to the smaller of the two boundary distances:

$$
0.1 \sigma_{i}^{\text {input }}<B_{i-/+}<\sigma_{i}^{\text {input }} \Rightarrow \sigma_{i}^{\text {initial }}=\min \left(B_{i-}, B_{i+}\right) .
$$

Case 2: When one or both of the boundary distances is smaller than a tenth of $\sigma_{\text {input }}$, we instead assume the likelihood is symmetric around the best-fit point, and we only compute steps in one direction (the one in which the distance to the boundary is the greatest), while mirroring the likelihood values to the other direction:

$$
B_{i-/+} \leq 0.1 \sigma_{i}^{\text {input }} \Rightarrow \sigma_{i}^{\text {initial }}=\min \left(\max \left(B_{i-}, B_{i+}\right), \sigma_{i}^{\text {input }}\right) .
$$

Once the steps have been settled in that way, the diagonal elements of the Fisher matrix are given by the numerical derivatives

$$
\mathbf{F}_{i i}=\frac{\partial^{2} \ln \mathcal{L}}{\partial p_{i}^{2}} \approx \frac{\ln \mathcal{L}\left(p_{i}+\Delta p_{i}\right)-2 \ln \mathcal{L}\left(p_{i}\right)+\ln \mathcal{L}\left(p_{i}-\Delta p_{i}\right)}{\Delta p_{i}^{2}}
$$

and the the off-diagonal ones by

$$
\mathbf{F}_{i j}=\frac{\partial^{2} \ln \mathcal{L}}{\partial p_{i} \partial p_{j}} \approx \frac{\ln \mathcal{L}\left(p_{i}+\Delta p_{i}, p_{j}+\Delta p_{j}\right)-\ln \mathcal{L}\left(p_{i}+\Delta p_{i}, p_{j}-\Delta p_{j}\right)-\ln \mathcal{L}\left(p_{i}-\Delta p_{i}, p_{j}+\Delta p_{j}\right)+\ln \mathcal{L}\left(p_{i}-\Delta p_{i}, p_{j}-\Delta p_{j}\right)}{4 \Delta p_{i} \Delta p_{j}} .
$$

Asymmetric steps. In cases 0 and 1, the code always uses symmetric steps, and in case 2 it postulates a symmetry of the likelihood. In some situations the user may find it beneficial to use instead some asymmetric steps to compute the Fisher matrix. This can be activated with the input flag:

- --fisher-asymmetric : allow for asymmetric steps (note: slows down computation) [default: False]

Then the "case 1" and "case 2" rules are replaced with some evaluations of the likelihood at $p_{i}+\Delta p_{+i}$ and $p_{i}-\Delta p_{-i}$, with $\Delta p_{+i}=\min \left(\sigma_{i}^{\text {input }}, B_{i+}\right)$ and $\Delta p_{-i}=\min \left(\sigma_{i}^{\text {input }}, B_{i-}\right)$. In that case the diagonal terms of the Fisher matrix are given by

$$
\frac{\partial^{2} \ln \mathcal{L}}{\partial p_{i}^{2}} \approx 2 \frac{\left(\frac{\Delta p_{-i}}{\Delta p_{+i}}\right) \ln \mathcal{L}\left(p_{i}+\Delta p_{+i}\right)-\left(\frac{\Delta p_{-i}}{\Delta p_{+i}}+1\right) \ln \mathcal{L}\left(p_{i}\right)+\ln \mathcal{L}\left(p_{i}-\Delta p_{-i}\right)}{\Delta p_{-i} \Delta p_{+i}+\Delta p_{-i}^{2}},
$$


and the off-diagonal ones by

$$
\begin{aligned}
\frac{\partial^{2} \ln \mathcal{L}}{\partial p_{i} \partial p_{j}} \approx & \left(\frac{\Delta p_{-j}^{2}}{\Delta p_{+j}}+\Delta p_{-j}\right)^{-1}\left(\frac{\Delta p_{-i}^{2}}{\Delta p_{+i}}+\Delta p_{-i}\right)^{-1} \\
& \times\left\{\left(\frac{\Delta p_{-j}}{\Delta p_{+j}}\right)^{2}\left[\left(\frac{\Delta p_{-i}}{\Delta p_{+i}}\right)^{2} \ln \mathcal{L}\left(p_{i}+\Delta p_{+i}, p_{j}+\Delta p_{+j}\right)-\ln \mathcal{L}\left(p_{i}-\Delta p_{-i}, p_{j}+\Delta p_{+j}\right)\right]\right. \\
& -\left(\frac{\Delta p_{-i}}{\Delta p_{+i}}\right)^{2} \ln \mathcal{L}\left(p_{i}+\Delta p_{+i}, p_{j}-\Delta p_{-j}\right)+\ln \mathcal{L}\left(p_{i}-\Delta p_{-i}, p_{j}-\Delta p_{-j}\right) \\
& +\left[\left(\frac{\Delta p_{-j}}{\Delta p_{+j}}\right)^{2}-1\right]\left(\ln \mathcal{L}\left(p_{i}-\Delta p_{-i}, p_{j}\right)-\left(\frac{\Delta p_{-i}}{\Delta p_{+i}}\right)^{2} \ln \mathcal{L}\left(p_{i}+\Delta p_{+i}, p_{j}\right)+\left[\left(\frac{\Delta p_{-i}}{\Delta p_{+i}}\right)^{2}-1\right] \ln \mathcal{L}\left(p_{i}, p_{j}\right)\right) \\
& \left.+\left[\left(\frac{\Delta p_{-i}}{\Delta p_{+i}}\right)^{2}-1\right]\left(\ln \mathcal{L}\left(p_{i}, p_{j}-\Delta p_{-j}\right)-\left(\frac{\Delta p_{-j}}{\Delta p_{+j}}\right)^{2} \ln \mathcal{L}\left(p_{i}, p_{j}+\Delta p_{+j}\right)\right)\right\} .
\end{aligned}
$$

\subsection{Efficient treatment of nuisance parameters}

Not counting the few intermediate steps necessary for the automatic determination of step sizes (which is typically around $2-4$ evaluations per parameter), the calculation of one Fisher matrix requires a number of likelihood evaluations equal to

$$
N_{\text {evaluations }}=1+2 N_{\text {params }}+4 \sum_{n=1}^{N_{\text {params }}-1} n,
$$

i..e. one in the best-fit point, two for each diagonal element and four for each off-diagonal element, where $N_{\text {params }}=$ $N_{\text {cosmo }}+N_{\text {nuisance }}$ is the total number of parameters, $N_{\text {cosmo }}$ is the number of cosmological parameters and $N_{\text {nuisance }}$ is the number of nuisance parameters. For a typical Planck run we have 6 cosmological parameters and 26 nuisance parameters, resulting in 2049 likelihood evaluations when the target $\Delta \chi^{2}$ is not iterated on.

The fact that varying only nuisance parameters does not require a call to the Boltzmann solver allows us to considerably optimize the computation. In MontePython, the routine calling the likelihoods always keeps a memory of the previous step (model parameters and cosmological observables). Therefore, if the likelihood is evaluated at a new point such that only nuisance parameters have changed, MontePython knows that the Boltzmann code should not be called again. To optimize the Fisher matrix calculation, we just need to arrange the $N_{\text {evaluations }}$ calls of the likelihood in a particular order minimizing the number of calls to the Boltzmann solver.

We loop over the parameters starting from the cosmological ones and ending with the nuisance ones.

For each parameter $p_{i}$, we first perform all the calculations involving the value $\left(p_{i}-\Delta p_{i}\right.$, i.e. $\mathcal{L}\left(p_{i}-\Delta p_{i}\right)$ for the diagonal element and $\mathcal{L}\left(p_{i}-\Delta p_{i}, p_{j} \pm \Delta p_{j}\right)$ (for each $\left.j>i\right)$ for the non-diagonal elements. Then we perform all the calculations involving the value $\left(p_{i}+\Delta p_{i}\right.$, i.e. $\mathcal{L}\left(p_{i}+\Delta p_{i}\right)$ for the diagonal element and $\mathcal{L}\left(p_{i}+\Delta p_{i}, p_{j} \pm \Delta p_{j}\right)$ (for each $j>i$ ) for the non-diagonal elements. In that way, the number of calls to the Boltzmann solver is drastically reduced to

$$
N_{\text {calls }}=1+4 N_{\text {cosmo }}+4 \sum_{n=1}^{N_{\text {cosmo }}-1} n
$$

corresponding to $N_{\text {calls }}=85$ for a typical Planck run (again without step iteration). This vastly reduces the computational time ${ }^{11}$

\footnotetext{
${ }^{11}$ Note that if the number of operation was not reordered in such a special way, we would still get some gain, but the number of calls would still be as large as

$$
N_{\text {calls }}=1+2 N_{\text {cosmo }}+4\left(\sum_{n=1}^{N_{\text {params }}-1} n-\sum_{m=1}^{N_{\text {nuisance }}-1} m .\right)
$$

This corresponds to $N_{\text {calls }}=697$ for a typical Planck run, without step iteration.
} 


\subsection{Plotting likelihood contours from inverse Fisher matrix}

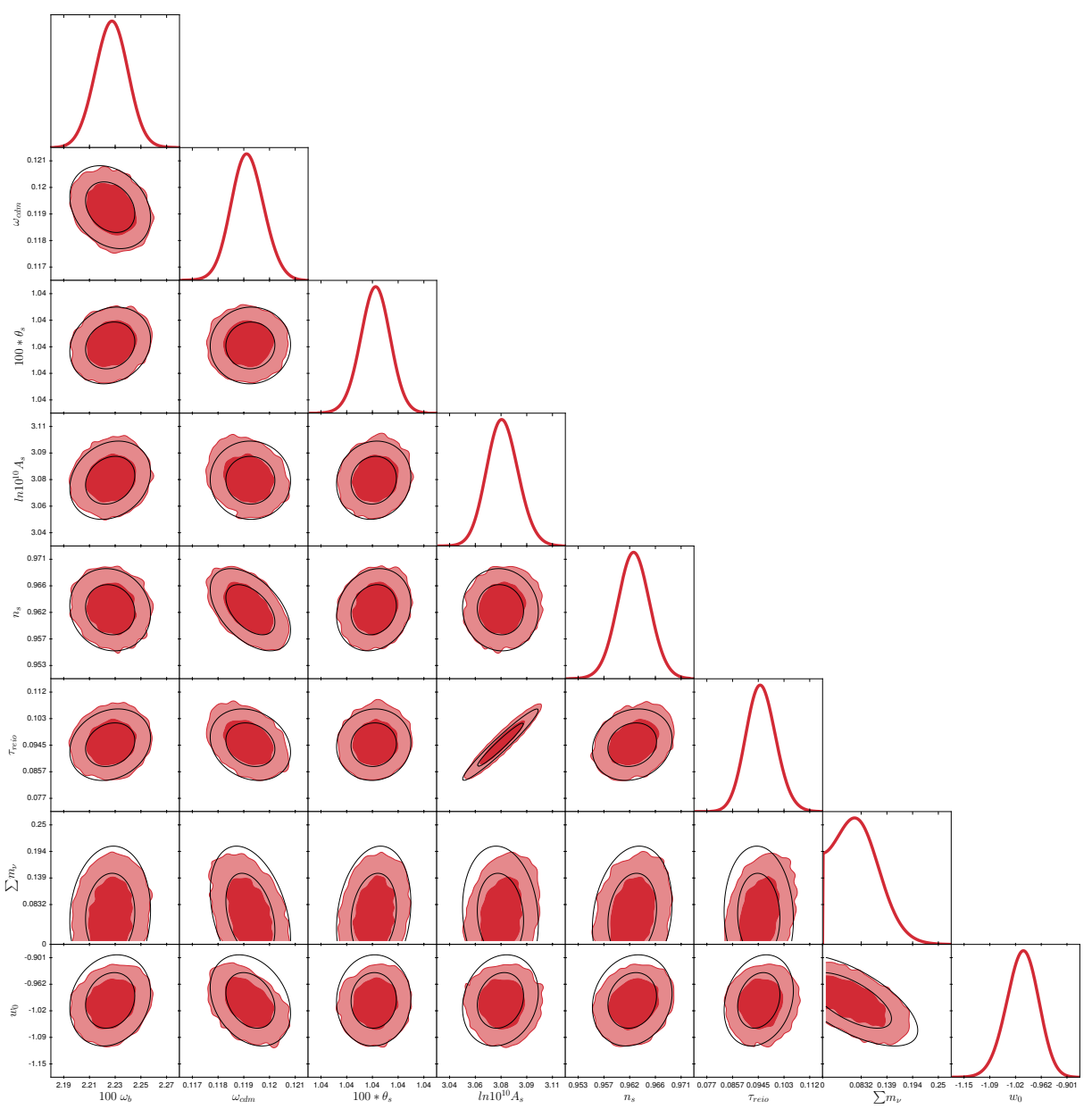

Figure 1: Confidence ellipses inferred from the inverse Fisher matrix (black lines) plotted on top of the 2d marginalized posterior distribution of a Metropolis-Hastings forecast. We fitted mock BAO data from the DESI survey combined with mock Planck data, assuming an 8 parameter cosmological model ( $v \mathrm{wCDM})$.

Having the Fisher matrix and its inverse, the user can easily write a small scripts to plot the ellipses corresponding to two-dimensional confidence levels in parameter space. This is not possible with MontePython, which can only do plots when some MCMC chains are present.

However, the MontePython plotting tools are useful for comparing the results of MCMC runs with the Gaussian posterior approximation given by the inverse Fisher matrix. For that purpose, one can analyse MontePython results with the usual info mode, adding just one input flag: --plot-fisher. Then the code will check whether a Fisher matrix has been computed and stored in the same directory as the chains that the user is trying to plot. If this is the case, the Fisher ellipses are drawn on top of the MCMC contours, like in figure 1

This figure shows a sensitivity forecast based on mock BAO data from the DESI survey combined with Planck data, for a cosmological model with massive neutrinos and dynamical dark energy ( $v$ wCDM, 8 free parameters). The Fisher matrix was actually computed before launching the chains, and its inverse was used as an input covariance matrix. The final MCMC contours prove that in this case, the Fisher approximation is excellent. This inverse Fisher matrix does not only provide a good proposal density for MCMC runs, it also gives excellent estimates of parameter bounds, and it could be substituted to the whole MCMC results.

One could be in a situation in which a Fisher matrix is first computed around a guess for the best-fit point, and then used to launch MCMC chains that will be centered on the true best-fit point (in the case of a Gaussian posterior). 
In the comparison plot, one may find that the Fisher ellipses have the right shape, but are offset with respect to the true best-fit point. In order to get a nicer plot, the MontePython user can use the input flag --center-fisher. This will automatically center the Fisher ellipses on the maximum likelihood point extracted from the MCMC chains, instead of using the central values read in the log.param file, even if the Fisher matrix was actually computed in that point.

\section{Illustration of performance}

In order to illustrate the performance of superupdate and the impact of using an inverse Fisher matrix as input covariance matrix, we have chosen a few data sets and cosmological models, and performed some fits with or without these different options. The comparison is especially interesting in the most difficult situations: large number of free parameters, small prior knowledge (i.e. poor guess for the input covariance matrix), etc.

\subsection{Forecasts with a small prior knowledge}

We first run some MCMC forecasts for the combination of mock BAO data from the DESI ${ }^{12}$ survey and Planck ${ }^{13}$ data. We use the mock DESI likelihood called fake_desi_vol (documented in Appendix D). For a forecast, we don't need to use real Planck data. We use instead a likelihood which simulates roughly the approximately of the Planck satellite, but uses some synthetic data corresponding to the Planck best-fit model. This likelihood is called fake_planck_realistic and is also documented in Appendix D.

Mock data: fake_planck_realistic, fake_desi_vol (see Appendix D)

Running time: 12 hours

\begin{tabular}{c|c|c|c|c} 
model & \# param. & $R-1:$ update & $R-1:$ superupdate & $R-1:$ superupdate + Fisher \\
\hline$\Lambda$ CDM & 6 & 0.030 & 0.015 & 0.013 \\
\hline$+\sum m_{v}+w_{0}$ & 8 & 0.036 & 0.022 & 0.018 \\
\hline$+N_{\text {eff }}+$ running & 10 & not converged & not converged & 0.040 \\
\hline$+\Omega_{k}$ & 11 & not converged & not converged & 0.048 \\
\hline$+w_{a}$ & 12 & not converged & not converged & 0.088
\end{tabular}

Running time: 48 hours

\begin{tabular}{c|c|c|c|c}
$\Lambda \mathrm{CDM}$ & 6 & 0.0035 & 0.0029 & 0.0019 \\
\hline$v w_{0} \mathrm{CDM}+N_{\text {eff }}+$ running & 10 & 0.014 & 0.0054 & 0.0038
\end{tabular}

Table 1: For mock data and several cosmological models, comparison of three sampling options, using the Gelman-Rubin convergence criterium. See text for details.

We fit these datasets with the minimal 6-parameter $\Lambda$ CDM model and with several extended models featuring up to 12 free parameters. These extensions are listed in the first column of Table 1 and include massive neutrinos, dynamical dark energy with a constant equation of state, extra relativistic degrees of freedom, a running of the primordial spectrum index, spatial curvature, and finally dynamical dark energy with a CPL parametrisation [25, 26].

We run MontePython in these different cases with the Metropolis-Hastings algorithm and three different methods:

- update [--update]: periodical update of the covariance matrix,

- superupdate [--superupdate]: additional adaptation of the jumping factor,

- superupdate + Fisher [first --method Fisher; then --superupdate]: same but starting from the inverse Fisher matrix computed by MontePython.

\footnotetext{
12 http://desi.lbl.gov

13 http://sci.esa.int/planck/
} 
For the update and superupdate runs, the proposal density is initialised as the "Planck 2015 covariance matrix", i.e. as the covariance matrix publicly distributed with the MontePython package, derived from the analysis of a wellconverged run based on the Planck 2015 likelihoods and assuming the 6-parameter $\Lambda$ CDM model. Also, in these two runs, the jumping factor is initially set to 2.4 (thus it remains equal to this value with the update method). For the Fisher matrix calculation, we pass to the code the exact best-fit model used to generate the mock data.

For each model and method, we launch the code with 8 chains, where each chain is running on 6 cores, using a total of 48 cores. After either 12 or 48 hours, we compute the worse [19] convergence criterium $(R-1)$ over all parameters, removing the initial $10-20 \%$ of each chain (depending on the duration of the burn-in phase, but always the same for a given combination of models and experiments).

The difficulty of these runs reside in the poor guess for the input covariance matrix. In the six parameter runs, the input covariance matrix is derived from Planck data alone, while the DESI BAO data is very constraining. This means the proposal density is much too wide initially, and needs to shrink to the small region allowed by DESI data. When adding extra parameters, the situation is even worse. For the extra parameters, the code does not rely on the input covariance matrix, but on the standard deviations written in the input file (for which we plug the Planck error bars). Therefore, the proposal density needs to learn both the correct order of magnitude for the jumps in these new directions, and the parameter correlations involving the extra parameters.

We find that, for the simplest models (6 and 8 parameters), all three methods successfully obtain at least a convergence of $R-1=0.03$, although superupdate and superupdate + Fisher perform better, obtaining an $R-1$ up to a factor 2 smaller. For the more complicated models (10,11 and 12 parameters), starting from a Fisher matrix and using superupdate makes a big difference, as only the runs starting from a Fisher matrix managed to obtain any level of convergence, when limiting ourselves to only 12 hours of runtime. However, if we allow for longer runtime (48 hours) the update and superupdate methods also manage to converge, thanks to periodic updates of the covariance matrix, with the superupdate and superupdate + Fisher runs showing a factor of 2.6 to 3.7 better convergence than update alone. Figure 2 explicitely shows why the jumping factor adaptation and the Fisher matrix calculation result in a very significant speed up for the convergence of this run.

\subsection{Current data}

For a comparison of the efficiency of our new methods using current data, we consider only the 6-parameter $\Lambda \mathrm{CDM}$ model, that we fit to two data sets: a small set with just Planck and BAO likelihoods, and a larger one including Large Scale Structure (LSS) likelihoods (galaxy clustering from SDSS and weak lensing from CFHTLens). More details are given in Table 2. We perform again some fits in three different ways (update, superupdate, superupdate+Fisher), exactly like in the previous section (i.e. starting update and superupdate from the "Planck 2015 covariance matrix" distributed with the code, and from a jumping factor 2.4). We use the same number of chains and cores as in the forecasts, and allow the chains to run for 12 or 48 hours.

Like in the previous section, these runs illustrate the case of starting from a bad guess for the proposal density, because the input covariance matrix takes only Planck into account and needs to shrink to the smaller region compatible with BAO and LSS data. There are other significant differences with respect to the runs of the previous section. First, when we use the "small" dataset, we have all the nuisance parameters of the Planck high- $\ell$ TT likelihood, which have strongly non-Gaussian posteriors and are correlated with each other. This means that the optimal jumping factor is significantly different from 2.4 (it is actually closer to 1.9). It also means that the Fisher matrix calculation is difficult, due to the large number of parameters, the non-Gaussianity of the likelihood with respect to some parameters, and the fact that we only have a poor approximation of the best-fit point in parameter space (we compute the Fisher matrix in the approximate best-fit extracted from the chains of an earlier run with Planck data only). With the extended data set, the code actually fails to obtain an invertible Fisher matrix with the full Planck TTTEEE + BAO + LSS data, so we had to switch to the Planck-lite TTTEEE likelihood in order to get rid of nuisance parameters.

The run with the small dataset shows the impact of the automatic jumping factor update: with superupdate, the code rapidly adapts the jumping factor to about 1.9 , while with update it remains stuck at 2.4 , leading to a small acceptance rate. Table 2 shows a gain in $(R-1)$ by a factor of two when using superupdate. However, this run also shows that using the inverse Fisher matrix is not always a good idea with current data and many non-Gaussian parameters, because the Fisher Matrix can be such a poor approximation of the likelihood (especially in the direction of the non-Gaussian nuisance parameters) that it is actually a worse input covariance matrix than the one derived from 

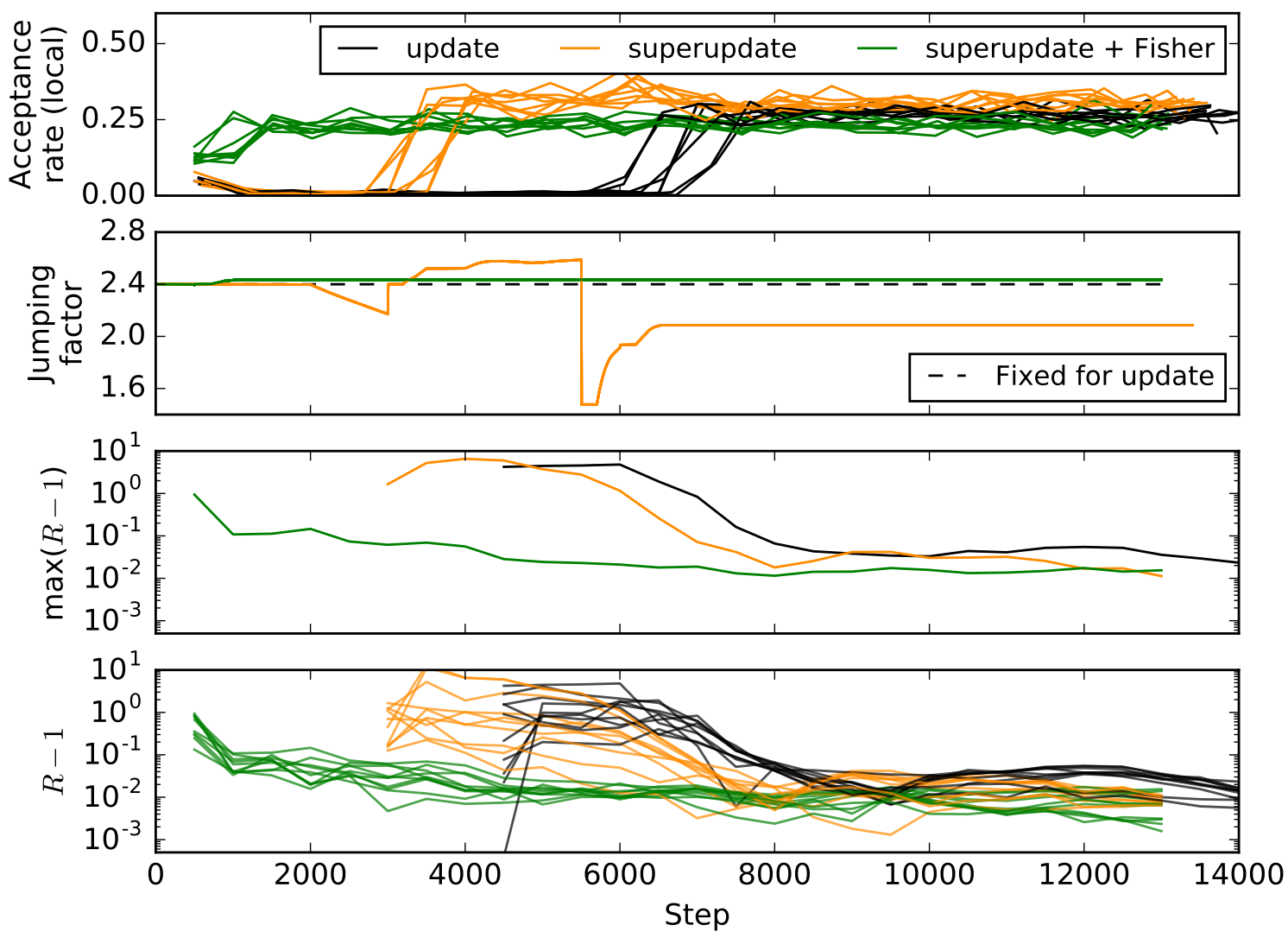

Figure 2: Evolution of the acceptance rate, jumping factor and convergence estimators for the last run from Table 1 (10 parameter model, mock Planck+DESI, 48h) using $U=50, S U=20, \mathrm{FPM}=10$, and thus $N_{\text {update }}=500$. The jumping factor information is updated at each step, and the information on $(R-1)$ every $N_{\text {update }}$ step (computed over the last $50 \%$ of the chain). This information can always be extracted from the code output. Instead, the local acceptance rate of the upper pannel was computed by post-processing the chains for the purpose of this plot, and was defined by averaging over about 500 steps (so this is slightly different from the quantity $\overline{a . r}$. used by the superupdate algorithms, which is only averaged over $S U \times$ FPM=200 steps). The "Fisher" run essentially catches the right covariance matrix, jumping factor and acceptance rate from the beginning. The "superupdate" run reduces its jumping factor in order to quickly accumulate many points and get a good covariance matrix estimate; once this is done, it increases the jumping factor to avoid a too big acceptance rate. Finally, the "update" run needs about 3500 more steps before entering into an efficient sampling regime with a good covariance matrix and acceptance rate, which corresponds to about 12 hours on our 48 cores: thus we can say that in this particular example, "superupdate" saved about 600 core-hours for a single run.

MCMC chains for a previous Planck-only run. Therefore, the preliminary Fisher calculation degrades the performance by a factor three compared to superupdate + the "Planck 2015 covariance matrix".

The run with the large dataset (but with the Planck-lite TTTEEE likelihood) shows instead the same trend as the forecasts: both superupdate and Fisher bring significant improvement, by up to a factor two in $(R-1)$.

These different situations bring us to the following conclusion, which match several other tests that we have performed and not included here:

- using superupdate is essentially always a good idea. The only situations in which one could consider sticking to update are the easiest ones, i.e. when a new run involves a dataset and a model so similar to a previous run that we already have an excellent knowledge of the covariance matrix and of the optimal jumping factor. In that case, update and superupdate are nearly equivalent, but in the most unlucky situations, superupdate could have a transitory phase during which the jumping factor would go away from the optimal value before 
Planck 2015 (highl TT, low $\ell$, lensing) + BAO (MGS, 6dFGS, LOWZ, CMASS)

Running time: 12 hours

\begin{tabular}{c|c|c|c|c} 
model & \# param. & $R-1:$ update & $R-1:$ superupdate & $R-1:$ superupdate + Fisher \\
\hline$\Lambda$ CDM & 6 & 0.019 & 0.0098 & 0.029
\end{tabular}

Planck 2015 (highl TTTEEE lite, low $\ell$, lensing) + BAO (MGS, 6dFGS, LOWZ, CMASS)

+ galaxy clustering (SDSS DR7 LRG), weak lensing (CFHTLenS)

\begin{tabular}{|c|c|c|c|c|}
\hline \multirow[b]{2}{*}{$\Lambda \mathrm{CDM}$} & \multicolumn{4}{|c|}{ Running time: 12 hours } \\
\hline & 6 & 0.042 & 0.032 & 0.018 \\
\hline & \multicolumn{4}{|c|}{ Running time: 48 hours } \\
\hline $\mathrm{CDM}$ & 6 & 0.0062 & 0.004 & 0.0038 \\
\hline
\end{tabular}

Table 2: For current data and the $\Lambda \mathrm{CDM}$ model, comparison of three sampling options, using the Gelman-Rubin convergence criterium. See text for details.

going back to it asymptotically, and would lose a bit in efficiency. This is normally marginal and we can safely recommend to use superupdate in all cases: then, depending on the "difficulty" of the run, the improvement will range from negligible to large.

- when there are many non-Gaussian parameters, such as the Planck nuisance parameters, the Fisher matrix computation often fails, and even when it does not fail, the inverse Fisher matrix is often a bad approximation for the proposal density, compared to any input covariance matrix that was inferred from chains with the same nuisance parameters. For example, this means that when using the full Planck high- $\ell$ likelihoods one should use the distributed "Planck 2015 covariance matrices", or one's own covariance matrices from previous runs, instead of the Fisher option. In almost all other cases, we found that computing and starting from the inverse Fisher matrix is a very powerful way to speed up convergence.

\section{Summary and conclusions}

We find that using superupdate and an inverse Fisher matrix as input covariance matrix reduces convergence time in most cases, and makes the process of obtaining convergence significantly simpler, due to much fewer trial and error runs being necessary.

The Fisher matrix computation is very quick, so we recommend that for forecasts (where the minimum is known) with a Metropolis-Hastings algorithm are preceded by a Fisher matrix computation, when an accurate covariance matrix is not available beforehand. Likewise, superupdate generally performs equal to or better than update alone, as it optimizes the acceptance rate, and we recommend its use for all Metropolis-Hastings runs.

We acknowledge that the calculation the Fisher matrix is not entirely robust in MontePython v3.0.0, since in difficult cases with many non-Gaussian parameters (such as the Planck nuisance parameters), the Fisher matrix found by the code can be non-invertible, due to numerical errors and/or a poor estimate of the best-fit parameter values. We expect that further progress can be made in the future in the minimization and Fisher algorithms. However, we have tested our new features in hundreds of runs (including the few cases detailed in section 4 ) and found them extremely convenient for saving CPU time.

\section{Acknowledgements}

We wish to thank Maria Archidiacono, Sébastien Clesse, Benedikt Schröer, Tim Sprenger and Thomas Tram for very useful input or feedback. This work was supported by the Deutsche Forschungsgemeinschaft through the graduate school "Particle and Astroparticle Physics in the Light of the LHC" and through the individual grant "Cosmological probes of dark matter properties". The runs for this paper have been using the facilities provided by the RWTH High Performance Computing cluster under the project rwth0113. 


\section{Appendix A. Extra parameterisations}

By design, any function in CLASS that has been incorporated into the CLASS python wrapper ${ }^{14}$ can be accessed directly from MontePython, without any additional coding required. However, sometimes it can be useful to define a specific parametrization within MontePython. This is easily done in the python/data.py module, where the MontePython input parameters can be intercepted and reshaped/renamed before being passed to CLASS. In the update_cosmo_arguments () function, all varying cosmological parameters are iterated through, and any additional parametrisations that are desired can be included by adding a simple if statement similar to existing ones.

MontePython includes several reparameterisation of this type. Some of them just deal with ordinary cosmological parameters, e.g.:

- if the parameter Omega_Lambda is used as a MontePython input parameter, instead of being passed to CLASS, it is used for defining $\mathrm{h}$ according to the rule $h=\sqrt{\left(\omega_{\mathrm{b}}+\omega_{\mathrm{cdm}}\right) /\left(1-\Omega_{\Lambda}\right)}$.

- if the parameter Omega_L is used as a MontePython input parameter, instead of being passed to CLASS, it is used for defining omega_cdm according to the rule $\omega_{\mathrm{cdm}}=\left(1-\Omega_{\Lambda}\right) h^{2}-\omega_{\mathrm{b}}$.

- if the parameter $\ln 10^{\wedge}\{10\} A_{-} s$ is used as a MontePython input parameter, instead of being passed to CLASS, it is used for defining A_s.

- if the parameter exp_m_2_tau_As ( $\left.\equiv e^{-2 \tau_{\text {reio }}} A_{s}\right)$ is used as a MontePython input parameter, instead of being passed to CLASS, it is used for defining A_s (assuming that tau_reio is also being used).

The code includes many other redefinitions related to isocurvature modes, neutrinos, dark energy, etc. Below we expand the discussion concerning a few of the implemented neutrino and Dark Energy reparameterisations. The user can easily extend the list of reparameterisation for her/his specific cases.

\section{Appendix A.1. Neutrino hierarchy}

MontePython can sample the total neutrino mass, with the individual neutrino masses arranged according to the Normal Hierarchy ( $\mathrm{NH}$, with two less massive and one more massive neutrino) or Inverted Hierarchy (IH, with one less massive and two more massive neutrinos).

The quantities passed to CLASS are the individual neutrino masses, but the quantity we are interested in sampling is the sum of neutrino masses. Formally, this is done using as a varying MontePython parameter M_tot_NH or M_tot_IH. Then, for each sampled value of the total neutrino mass $\left(M_{v}\right)$, the individual neutrino masses $\left(m_{i}\right)$ are calculated by solving the system of equations (see e.g. [27])

$$
\begin{aligned}
& M_{v}=m_{1}+m_{2}+m_{3}, \\
& \Delta m_{\mathrm{atm}}^{2}=m_{3}^{2}-m_{\ell}^{2}, \\
& \Delta m_{\mathrm{sol}}^{2}=m_{2}^{2}-m_{1}^{2},
\end{aligned}
$$

where $\ell$ is 1 for $\mathrm{NH}$ and 2 for $\mathrm{IH}$, and $\Delta m_{\mathrm{atm}}^{2}$ and $\Delta m_{\mathrm{sol}}^{2}$ are the current central values of the mass splittings obtained from neutrino oscillation experiments [28] (for a more recent study see e.g. [29])

$$
\begin{array}{ll}
\text { NH: } & \Delta m_{\text {atm }}^{2}=2.524 \times 10^{-3} \mathrm{eV}^{2}, \\
& \Delta m_{\text {sol }}^{2}=7.50 \times 10^{-5} \mathrm{eV}^{2}, \\
\text { IH: } & \Delta m_{\text {atm }}^{2}=-2.514 \times 10^{-3} \mathrm{eV}^{2}, \\
& \Delta m_{\text {sol }}^{2}=7.50 \times 10^{-5} \mathrm{eV}^{2} .
\end{array}
$$

\footnotetext{
${ }^{14}$ Note that "incorporating" a new CLASS parameter in the wrapper just consists of adding one line in python/cclassy.pxd with just a declaration of this parameter (e.g. double my_param). The declaration must be done within the structure to which the parameter belongs. Incorporating a new CLASS function also boils down to declaring it in this file. New coding in the file python/classy. pyx is only required when one wants to create a new function specific to the wrapper itself, rather than just interfacing a CLASS function.
} 
Additionally, the three parameters $N \_u r=0.00641, N \_n c d m=3$ and T_ncdm $=$ '0.71611,0.71611,0.71611' should be fixed as cosmo_arguments, to reflect the fact that have three distinct standard active neutrino species and no extra relativistic degrees of freedom (unless one is studying a scenario with extra relativistic relics, in which case N_ur should be varied). Note that T_ncdm gives, for each species, the temperature of the neutrinos in units of photon temperature. In reality, the standard neutrinos distribution contains slightly non-thermal distribution, while by default CLASS will treat them as thermal species. The price to pay is to have a temperature ratio adjusted to 0.71611 in order to get the right neutrino density in the non-relativistic regime, and a small contribution to $N_{-}$ur in order to get the right density in the relativistic one. This is why with three massive species we advise to take N_ur $=0.00641$ instead of N_ur=0, but this correction is anyway well below the sensitivity of current experiments.

\section{Appendix A.2. Degenerate massive v's and varying $N_{\mathrm{eff}}$}

In addition to arranging the mass of the neutrinos in a neutrino hierarchy, it is possible to sample the total neutrino mass for a case with three massive neutrinos with degenerate mass. Although not a realistic scenario, it is often sufficient to use three degenerate neutrinos (see e.g. [30]), speeding up computations in the Boltzmann solver.

This is done via the input parameter M_tot, remembering to specify the cosmo_arguments from before, but this time with only one type of neutrino species, $N \_u r=0.00641, N \_n c d m=1$ and $T \_n c d m=0.71611$, and instead specifying the degeneracy of the neutrino species, deg_ncdm=3. The total neutrino mass is then simply divided by the number of massive neutrino species and the resulting particle mass is passed to CLASS.

Additionally, this allows for varying the effective number of relativistic species, $\mathrm{N}_{\mathrm{eff}}$, by using the degeneracy of neutrino species, deg_ncdm, as a varying cosmological parameter instead of a fixed quantity.

For completeness, it is possible to use only one or two degenerate massive neutrinos and the rest massless, but this has been shown to be slightly inaccurate for the precision of current experiments [31].

\section{Appendix A.3. Dynamical dark energy}

Many phenomenological dark energy models can be treated using the fluid sector of CLASS, which has several free parameters labelled as _fld. By default, this sector uses the PPF parameterisation [32], although real fluid equation can be restored by setting use_ppf to 'no' in the cosmo_arguments.

In principle, the dynamical dark energy equation of state parameters w0_fld and wa_fld, with a CPL parameterisation [25, 26] defined through $w(a)=p_{D E} / \rho_{D E}=w_{0}+w_{a}\left(1-a / a_{0}\right)$ (where, as usual, $p$ is the pressure, $\rho$ is the density, and $a$ is the scale factor), can be passed directly to CLASS. However, it may be useful to sample the quantity $w_{0}+w_{a}$ (implemented as w0wa) and $w_{0}$ (implemented w0_fld), in order to restrict the parameter space of $w_{0}+w_{a}$ to only negative values (as in e.g. [33]).

\section{Appendix A.4. Sterile v parametrization}

A final example of how a specific parameterisation can be introduced in MontePython is sterile neutrinos. In addition to degenerate massive neutrinos, one may wish to sample the sterile neutrino mass and the contribution of sterile neutrinos to $\mathrm{N}_{\text {eff }}$, while avoiding the region of parameter space where the sterile neutrino mass becomes arbitrarily large and the contribution to $\mathrm{N}_{\text {eff }}$ becomes arbitrarily small (see Fig. 32. of [34]).

For this we defined the effective sterile neutrino mass $m_{s, \text { eff }} \equiv m_{s} \Delta N_{s}$ as a possible varying cosmological parameter (m_s_eff), that should be used along with the parameter deg_ncdm_-2 standing for the contribution of sterile neutrinos to $\mathrm{N}_{\mathrm{eff}}$. This case is a bit more complicated than the others, as, in addition to setting $\mathrm{N} \_\mathrm{ncdm}=2$ and $\mathrm{T} \_\mathrm{ncdm}=$ ' $0.71611,0.71611$ ', we also need to set the degeneracy of normal neutrinos deg_ncdm_-1 as a 'phantom' varying cosmological parameter, but with the parameter fixed to 3. The mass of the degenerate active neutrino species can also be varied as $\mathrm{m}_{-} \mathrm{ncdm} \mathrm{d}_{-}$. This means the output of the chains will be the mass of a single active neutrino, rather than the sum, but of course we know that in this case $M_{v}=$ m_ncdm $\_1 \times$ deg_ncdm $\_1=3$ m $\_$ncdm $\_1$.

The effective sterile neutrino mass is then converted to physical sterile neutrino mass within the data.py module, in the function update_cosmo_arguments (), by dividing with $\Delta N_{s}$ (assuming that this is the ncdm species number 2 and that it is Dodelson-Widrow-like, i.e with the same temperature as active neutrinos). It is finally passed to CLASS along with the other neutrino masses. 


\section{Appendix B. Sampling options}

MontePython has the following general sampling options

- --method : sampling method (MH, NS, CH, IS, Der, Fisher) [default: MH]

which refer respectively to Metropolis-Hastings, Nested Sampling (= MultiNest), Cosmo Hammer (= emcee), Importance Sampling, Derived (= reprocessing the chains to add columns with extra derived parameters requiring a new CLASS run for each model), and Fisher.

- - T : sample from the probability distribution $P^{1 / T}$ instead of P [default: 1.0$]$

Options for Metropolis-Hastings and variants

- --method MH : Metropolis-Hastings sampling [default: $\mathrm{MH}$ ]

- --update : proposal distribution update frequency in number of cycles [default: 50]

- --superupdate : also adapt jumping factor. Adaptation delay in number of cycles [default: 0] (i.e. deactivated by default. Recommended: 20)

- --superupdate-ar : target local acceptance rate [default: 0.26 ]

- --superupdate-ar-tol : tolerance for local acceptance rate [default: 0.01]

- --adaptive : running adaptation of covariance matrix and jumping factor (note: only suitable for single chain runs) [default: 0]

- --adaptive-ts : starting step for adapting the jumping factor [default: 1000]

- $-f$ : jumping factor [default: 2.4 ]

- --minimize : attempt to re-evaluate starting point using a $\chi^{2}$ minimization algorithm [by default uses SLSQP via numpy.optiminize.minimize(), can be changed in sampler.py function get minimum()]

- --minimize-tol : tolerance for minimization [default: $10^{-5}$ ]

Fisher matrix options

- --method Fisher : compute a Fisher matrix [default: MH]

- --fisher-asymmetric : allow for asymmetric steps (note: slows down computation) [default: False]

- --fisher-step-it : number of step iterations attempted [default: 10]

- --fisher-delta : target $\Delta \ln \mathcal{L}$ value for step iteration [default: 0.1 ]

- --fisher-tol : tolerance for $\Delta \ln \mathcal{L}$ (note: decreasing slows down computation) [default: 0.05]

- --fisher-sym-lkl : cut-off for switching to symmetric likelihood assumption in units of $\sigma$. Relevant when parameter space boundaries are close to the central value [default: 0.1 ]

MontePython also supports sampling with MultiNest (--method NS) [35-37] via a python wrapper [38] and emcee [39.41] via CosmoHammer (--method $\mathrm{CH}$ ) [41]. For these sampling options we refer to the official documentation of those codes. 


\section{Appendix C. Analyze and plotting options}

The range of plotting options and the general presentation of the plots has been significantly improved in MontePython v3.0.0. We should, however, point out that the user is free to use other plotting tools, if she/he prefers. In particular, the MontePython output is fully compatible with Antony Lewis's GetDist ${ }^{15}$. Note that MontePython writes in each output directory a file in the . paramnames format just for this purpose. GetDist has some very advanced plotting functionalities and a very nice graphical interface. However, the user will benefit from a few advantages when using the MontePython analyzing and plotting tools, such as: automatically evaluating the burn-in phase; automatically eliminating the non-Markovian part of the chains; and automatically reading information regarding the parameter names, ranges and scalings in the log. param file.

\section{Appendix C.1. Chain analysis}

When analyzing the chains, MontePython eliminates automatically the burn-in phase at the beginning of each chain, before applying additional cuts that can be customised with the options listed below. The burn-in phase of each chain is defined as: all the first points in the chains until an effective $\chi^{2}$ value smaller than $\chi_{\min }^{2}+6$ was reached for the first time. This number of 6 can be adjusted manually (it is equal to 2 LOG_LKL_CUTOFF, where LOG_LKL_CUTOFF is a parameter set in montepython/analyze.py, with a default value of 3). For runs in which a good estimate of the best-fit model was passed in input (with the option $-\mathrm{b}\langle\mathrm{xxx}\rangle$. bestfit), the burn-in phase defined in this way may not exist at all.

Additionally, MontePython has the following options for analyzing chains (thus they should be written after the command line python montepyhton/MontePython.py info):

- --keep-non-markovian : keep the non-Markovian part of the chains [default: False].

- --keep-fraction : pass a decimal fraction, e.g. 0.8 to keep the last $80 \%$ of the part of the chains that remain after the burn-in removal (note: redundant if non-Markovian points are discarded) [default: 1.0]

- --want-covmat : compute a covariance matrix based on the chains (note: this will overwrite the one produced by --update) [default: False]

- --bins : the number of bins for computing histograms [default: 20]

- $-\mathrm{T}$ : raise posteriors to the power $\mathrm{T}$ [default: 1.0$]$

- --silent : do not write any standard output (useful when running on clusters) [default: False]

- --minimal : use this flag to avoid computing posteriors, confidence limits and plots. The code just analyses the chains and outputs the files containing the convergence statistics, the best-fit parameters, and possibly the covariance matrix if --want-covmat is on [default: False]

Updating the proposal distribution or jumping parameter means that all prior steps in the chain are no longer Markovian, i.e. that each step should not depend on any prior steps. However, by using appropriate criteria for stopping adaptation of the jumping parameter and proposal distribution, and only including all steps after this point in our final analysis, we can ensure that our process was still Markovian. This is automatically done, but can be disabled with the command --keep-non-markovian, especially in slowly converging cases, when the user struggles to get a good covariance matrix that would stop the updating process, and wants to see some approximate results anyway. Although the burn-in phase is always removed, if non-Markovian steps are included the user may want to use the command--keep-fraction <number> in order to remove the first part of the chain.

\footnotetext{
15 http://getdist.readthedocs.io/en/latest/
} 


\section{Appendix C.2. Basic plotting}

The most basic plotting features are implemented as command line options (but many of them can also be passed through an input customisation file, as we shall in Appendix C.3):

- --no-plot : disable plotting [default: False]

- --no-plot-2d : only plot 1d posterior distributions [default: False]

- --all : output all individual 2D subplots and histogram files as separate files

- --ext : format and extension of the plot files (pdf, eps, png) [default: pdf]

- --no-mean : in 1D plot, do not plot the "mean likelihood" as dashed lines, only plot the posteriors as solid lines [default: False]

- --contours-only : line contours instead of filled contours [default: False]

- --posterior-smoothing : smoothing scheme for 1D posteriors: 0 means no smoothing, 1 means cubic interpolation, $n>1$ means fitting $\ln (\mathcal{P})$ with a polynomial of order $n$ [default: 5]

- --interpolation-smoothing : for 2D contours only, interpolation factor for getting a finer histogram before applying Gaussian smoothing and getting contours; 1 means no interpolation, increase for finer curves [default: 4]

- --gaussian-smoothing : for 2D contours only, width of Gaussian smoothing applied to histogram before getting contours, in units of bin size; increase for smoother contours, decrease for more exact results [default: $0.5]$

- --short-title-1d : short 1D plot titles. Remove mean and confidence limits above each 1D plots. [default: False]

- --num-columns-1d : for 1D plots, number of plots per horizontal raw; if 'None' this is set automatically (trying to approach a square plot) [default: None]

- --fontsize desired fontsize [default: 16]

- --ticksize desired ticksize [default: 14]

- --line-width set line width [default: 4]

- --decimal number of decimal places on ticks [default to 3]

- --ticknumber number of ticks on each axis [default to 3]

- --legend-style specify the style of the legend, to choose from 'sides" or 'top" [default: sides]

When an Inverse Fisher matrix has been computed --method Fisher, the Fisher ellipses can be plotted on top of MCMC contours using the plotting options:

- --plot-fisher : plot inverse Fisher matrix contours [default: False]

- --center-fisher : centers Fisher ellipses on the parameters extracted from the best-fit model found in the chains, instead of the central starting values found in the input file [default: False] 


\section{Appendix C.3. More advanced plot customisation}

Further options for customizing plots can be passed through a file with extension .plot called with the option --extra. All functionalities are mentioned in the example file plot_files/example.plot that the user would call with the plotting option --extra plot_files/example.plot. Although this file is self-explanatory, we list here the main functionalities provided by the use of .plot files. Several options have been present since the first release of MontePython:

- on-the-fly redefinition of the chain parameters with a simple syntax. For instance, if you know that there is a parameter called A and one called B, you can in principle replace the numbers in the column A by the result of any algebraic operation involving $\mathrm{A}$ alone, or $\mathrm{A}$ and $\mathrm{B}$, or even more parameters, like e.g. $\mathrm{A}+3 \mathrm{~A} / \mathrm{B}$. The file plot_files/example.plot provides the following example:

$$
\text { info.redefine }=\left\{\text { 'omega_cdm' }^{\prime} \quad,(0.01 * \text { omega_b+omega_cdm }) /(\mathrm{HO} / 100 .) * * 2{ }^{\prime}\right\}
$$

In this example, the code takes the numbers in the column omega_b and first multiplies them by 0.01 , knowing that in the chains, $\omega_{b}$ was rescaled by 100 (this actually depends on what the user wrote in the input file). Thus, $0.01 *$ omega_b is the true $\omega_{\mathrm{b}}$, and (0.01*omega_b+omega_cdm)/(H0/100.) **2 is in fact $\Omega_{\mathrm{m}}$. With the above command, each value of $\omega_{\mathrm{cdm}}$ is replaced on-the-fly by $\Omega_{\mathrm{m}}$ when the chains are read. The next necessary step is to change the name of the parameter for this column from omega_cdm to Omega m, which can be done by the next functionality.

- redefinition of parameter name, for the purpose of redefinitions or making the parameter name better readable by the LaTeX routines of the plotting algorithm, e.g.

info.to_change $=\{$ 'omega_cdm': '\$Omega_ $\backslash$ mathrm $\{\mathrm{m}\} \$$ ' $\}$

will replace omega_cdm with Omega_m. Note that, for the purpose of getting a nice LaTeX format, MontePython already does several basic operations automatically, like identifying greek letters, subscripts and superscripts. Hence, at the time of producing a plot label, it would automatically convert omega_cdm into $\$ \backslash$ omega_ $\{c d m\} \$$. The functionality info.to_change is useful in order to further customise the LaTeX formatting.

- redefine the overall rescaling factor when the one from the input file is not optimal (scaling factors are useful e.g. to get rid of powers of ten in the plot captions, for very small or large parameters). This is done with the syntax info.new_scales $=\left\{{ }^{\prime} A^{\prime}: 100\right\}$.

- specify the list of parameters to be plotted (taking into account the new names, if there were name redefinitions). This is done with the info.to_plot $=[\ldots]$ syntax, which is very useful e.g. for getting rid of nuisance parameters in the $1 \mathrm{D}$ and $2 \mathrm{D}$ plots.

The new functionalities in MontePython v3.0.0 are:

- parameters to control the legends: info.plot_legend_1d, info.plot_legend_2d, info.legendnames (see plot_files/example.plot for details).

- parameters to control the colors: info.MP_color_cycle, info.MP_color, info.alphas (see plot_files/example.plot for details).

- these lines simply overwrite the value of some parameters defined previously by the code within the python class info. Many other such lines can be added there, for instance info.ticknumber $=5$, etc. Thus some of the options described previously as command line options can also be passed here, as lines of python.

- sometimes, the user would like to add some extra lines of python code in the plotting script, in order to further customise 1D or 2D plots, e.g. with vertical or horizontal lines, bands, arrows, labels, etc.. Usually, these lines are meant for only specific 1D or 2D plots. One can now achieve this by writing a few extra lines of python code in little files with a .py extension, which will be read and executed before finalizing the relevant plots. If they start with appropriate if statements, they will only be taken into account when plotting specific parameters. Some self-explanatory examples are provided together with the code in the files plot_files/example.plot, add_h_contour.py, and add_sigma8_Omegam_contour.py. 


\section{Appendix D. Likelihoods}

Below is a comprehensive list of the likelihoods in the MontePython v3.0.0 package, as well as references to the paper(s) that should be cited when used (i.e. either where the likelihood was published and/or first used with MontePython). We recall that it is easy to modify these likelihoods or to create new ones. Some guidelines are given in the MontePython online documentation ${ }^{16}$, in the section "Existing likelihoods, and how to create new ones". The column LU (Last Updated) shows the version number of the last modification.

In the column D (Dependencies), SC stands for self-contained; D means that some external data files must be downloaded; W means that we provide a wrapper to some external likelihood code that must be downloaded together with some data (as e.g. for Planck likelihoods); M means that this likelihood will automatically generate its own mock data, unless it has already been generated by a previous run. In the cases D, W, M, if you run the likelihood before downloading the required external files or before having created mock data, a self-explanatory message will tell you where to download from or what to do.

\begin{tabular}{|c|c|c|c|c|c|}
\hline name & $\begin{array}{l}\text { Current data likelihoo } \\
\text { description }\end{array}$ & type & LU & $\mathrm{D}$ & reference(s) \\
\hline acbar & ACBAR 2017 & CMB & 1.0 & $\mathrm{SC}$ & [42] \\
\hline bao & $\begin{array}{c}\text { 6dFGS } \\
\text { BOSS DR9, } \\
\text { SDSS DR7 }\end{array}$ & $\mathrm{BAO}$ & 1.1 & $\mathrm{SC}$ & $\begin{array}{l}43 \\
44 \\
45 \\
45 \\
4\end{array}$ \\
\hline bao_known_rs & $\begin{array}{c}\text { same as bao assuming } \\
\text { known sound horizon value }\end{array}$ & $\mathrm{BAO}$ & 1.1 & $\mathrm{SC}$ & [46] \\
\hline bao_angular & $\begin{array}{c}\text { angular 2-point } \\
\text { correlation function } \\
\text { SDSS DR7: LRG } \\
\text { BOSS DR10\&11: CMASS } \\
\text { BOSS DR12: QSO }\end{array}$ & $\mathrm{BAO}$ & 3.0 & $\mathrm{SC}$ & $\begin{array}{l}{[47]} \\
{[48]} \\
{[49]} \\
{[50]} \\
{[51}\end{array}$ \\
\hline bao_boss & $\begin{array}{c}\text { 6dFGS, } \\
\text { BOSS DR10\&11: } \\
\text { LOWZ, CMASS, } \\
\text { SDSS DR7: MGS }\end{array}$ & $\mathrm{BAO}$ & 2.0 & $\mathrm{SC}$ & $\begin{array}{l}{[43} \\
{[52]} \\
{[45} \\
4\end{array}$ \\
\hline bao_boss_aniso & BOSS DR10\&11: CMASS & $\mathrm{BAO}$ & 2.0 & $\mathrm{SC}$ & [52] \\
\hline bao_boss_aniso_gauss_approx & BOSS DR10\&11: CMASS & BAO & 2.0 & $\mathrm{SC}$ & [52] \\
\hline bao_boss_dr12 & $\begin{array}{c}\text { BOSS DR12: } \\
\text { LOWZ \& CMASS }\end{array}$ & $\mathrm{BAO}$ & 3.0 & $\mathrm{SC}$ & $\begin{array}{l}{[53]} \\
{[54]} \\
\end{array}$ \\
\hline bao_fs_boss_dr12 & $\begin{array}{c}\text { BOSS DR12: } \\
\text { LOWZ \& CMASS }\end{array}$ & $\mathrm{BAO}+\mathrm{RSD}$ & 3.0 & $\mathrm{SC}$ & $\begin{array}{l}{[53} \\
{[54} \\
54\end{array}$ \\
\hline bao_smallz_2014 & $\begin{array}{c}\text { 6dFGS, } \\
\text { SDSS DR7: MGS }\end{array}$ & $\mathrm{BAO}$ & 3.0 & $\mathrm{SC}$ & $\begin{array}{l}{[43]} \\
{[45]}\end{array}$ \\
\hline bicep & BICEP & CMB & 1.0 & $\mathrm{SC}$ & [55] \\
\hline bicep2 & BICEP2 & CMB & 2.0 & $\mathrm{SC}$ & [56] \\
\hline BK14 & Bicep-Keck-Planck 2014 & CMB & 3.0 & $\mathrm{D}$ & [57] \\
\hline BK14priors & priors for the latter & CMB & 3.0 & $\mathrm{D}$ & [57] \\
\hline boomerang & BOOMERanG & CMB & 1.0 & $\mathrm{SC}$ & {$[58$} \\
\hline cbi & CBIpol & CMB & 1.0 & $\mathrm{SC}$ & [59] \\
\hline CFHTLens & CFHTLens as $\Omega_{m}^{\alpha} \sigma_{8}$ prior & Weak Lens. & 2.1 & $\mathrm{SC}$ & 60 \\
\hline CFHTLens_correlation & full CFHTLens correlation & Weak Lens. & 2.2 & $\mathrm{SC}$ & [60] \\
\hline
\end{tabular}

Table D.3: Current data likelihoods (letters a-c)

\footnotetext{
${ }^{16}$ http://monte-python.readthedocs.io
} 


\begin{tabular}{|c|c|c|c|c|c|}
\hline name & description & type & LU & $\mathrm{D}$ & reference(s) \\
\hline clik_wmap_full & $\begin{array}{c}\text { WMAP 7yr } \\
\text { (through Planck wrapper) }\end{array}$ & CMB & 1.2 & $\overline{\mathrm{W}}$ & [61] \\
\hline clik_wmap_lowl & WMAP 7yr: low $\ell$ & CMB & 1.2 & W & [61] \\
\hline cosmic_clocks_2016 & cosmic clocks & $H(z)$ & 3.0 & $\mathrm{SC}$ & [62] \\
\hline cosmic_clocks_BC03 & cosmic clocks & $H(z)$ & 2.1 & $\mathrm{SC}$ & [63] \\
\hline cosmic_clocks_MaStro & cosmic clocks & $H(z)$ & 2.1 & SC & [63] \\
\hline cosmic_clocks_BC03_all & cosmic clocks & $H(z)$ & 2.1 & $\mathrm{SC}$ & $\begin{array}{l}{[63]} \\
64 \mid \\
{[64}\end{array}$ \\
\hline da_rec & prior on angular diameter distance & $d_{A}\left(z_{\text {rec }}\right)$ & 1.1 & SC & [46] \\
\hline gunn_peterson & constraints on reionization history & $x_{e}(z)$ & 1.0 & SC & [66] \\
\hline hst & Hubble Space Telescope & $H_{0}$ prior & 3.0 & $\mathrm{SC}$ & [67] \\
\hline igm_temperature & constrains on baryon temperature & $T_{b}(z)$ & 1.0 & SC & [68] \\
\hline ISW & NVSS,2MPZ,WI×SC,SDSS/Planck & ISW & 3.0 & SC & [69] \\
\hline JLA & full JLA likelihood & Supernovae & 2.1 & $\mathrm{D}$ & [70] \\
\hline JLA_simple & simplified JLA likelihood & Supernovae & 2.1 & $\mathrm{D}$ & [70] \\
\hline kids450_qe_likelihood_public & KiDS-450 & Weak lensing & 3.0 & $\mathrm{D}$ & [71] \\
\hline lowlike & Planck 2013 + WMAP 9: low- $\ell$ & CMB & 1.2 & W & [72] \\
\hline Planck_actspt & ACT 2013, SPT 2011 & $\mathrm{CMB}$ & 2.0 & $\mathrm{~W}$ & [73] \\
\hline Planck_highl & Planck 2015: TT high $\ell$ & $\mathrm{CMB}$ & 2.2 & $\mathrm{~W}$ & [75] \\
\hline Planck_highl_lite & Planck 2015: TT high $\ell$ lite & CMB & 2.2 & W & [75] \\
\hline Planck_highl_TTTEEE & Planck 2015: TTTEEE high $\ell$ & $\mathrm{CMB}$ & 2.2 & W & [75] \\
\hline Planck_highl_TTTEEE_lite & Planck 2015: TTTEEE high $\ell$ lite & CMB & 3.0 & $\mathrm{~W}$ & [75] \\
\hline Planck_lensing & Planck 2015: lensing & CMB lensing & 2.2 & $\mathrm{~W}$ & [76] \\
\hline Planck_lowl & Planck 2015: TTTEEE low $\ell$ & $\mathrm{CMB}$ & 2.2 & $\mathrm{~W}$ & [75] \\
\hline Planck_SZ & $\begin{array}{l}\text { Planck 2015: SZ cluster counts } \\
\text { as } \Omega_{m}^{\alpha} \sigma_{8} \text { prior }\end{array}$ & Cluster Count & 2.2 & SC & [77] \\
\hline polarbear & Polarbear & CMB & 2.1 & $\mathrm{SC}$ & [78] \\
\hline quad & QUAD DR3 & CMB & 1.0 & SC & [79] \\
\hline sdss_lrgDR4 & SDSS DR4: LRG & Galaxy Clust. & 3.0 & SC & [80] \\
\hline sdss_lrgDR7 & SDSS DR7: LRG & Galaxy Clust. & 3.0 & SC & \begin{tabular}{|l|}
$81]$ \\
$54]$ \\
\end{tabular} \\
\hline simlow & from Planck 2016: TTTEEE low $\ell$ & $\tau_{\text {reio }}$ prior & 3.0 & SC & $\frac{\mid 82]}{[83]}$ \\
\hline sn & Union2 & Supernovae & 1.0 & SC & [84] \\
\hline spt & SPT DR1 & CMB & 1.0 & SC & [85] \\
\hline spt_2500 & SPT DR $1, \ell \leq 2500$ & CMB & 1.0 & SC & [85] \\
\hline timedelay & quasar time delays & Time Delay & 1.1 & SC & [86] \\
\hline WiggleZ & WiggleZ power spectrum & Galaxy Clust. & 2.0 & SC & [87] \\
\hline WiggleZ_bao & WiggleZ BAO & $\mathrm{BAO}$ & 2.1 & $\mathrm{SC}$ & [88] \\
\hline wmap & WMAP 7yr (own wrapper) & $\mathrm{CMB}$ & 1.0 & $\mathrm{D}$ & [61] \\
\hline wmap_9yr & WMAP 9yr (own wrapper) & CMB & 1.2 & $\mathrm{D}$ & [89] \\
\hline
\end{tabular}

Table D.4: Current data likelihoods (letters c-z) 
Forecast likelihoods

\begin{tabular}{l|c|c|c|c|c} 
name & description & type & LU & $\mathrm{D}$ & reference(s) \\
\hline \hline core_m5 & CORE M5 ESA proposal & CMB & 3.0 & $\mathrm{M}$ & {$[30]$} \\
\hline euclid_lensing & Euclid & Weak Lensing & $3.0^{*}$ & $\mathrm{M}$ & {$[90]$} \\
\hline euclid_pk & Euclid & Galaxy Clust. & $3.0^{*}$ & $\mathrm{M}$ & {$[91]$} \\
& & & & & {$[92]$} \\
\hline fake_desi & DESI & BAO: $d_{A} / r_{s}$ & 3.0 & $\mathrm{M}$ & {$[30]$} \\
\hline fake_desi_euclid_bao & best from DESI + Euclid & BAO & 3.0 & $\mathrm{M}$ & {$[93]$} \\
\hline fake_desi_vol & DESI & BAO: $r_{s} / d_{V}$ & 3.0 & $\mathrm{M}$ & {$[94]$} \\
\hline fake_planck_bluebook & Planck 2015 est.: TTTEEE & CMB & 2.0 & $\mathrm{M}$ & {$[95]$} \\
\hline fake_planck_realistic & Planck 2018 est.: TTTEEE $\phi \phi$ & CMB & 3.0 & $\mathrm{M}$ & {$[30]$} \\
\hline litebird & LiteBIRD est. & CMB & 3.0 & $\mathrm{M}$ & {$[30]$} \\
\hline ska1_IM_band1 & SKA1 band 1 & $21 \mathrm{~cm}$ Int. Map. & $3.0^{*}$ & $\mathrm{M}$ & {$[92]$} \\
\hline ska1_IM_band2 & SKA1 band 2 & $21 \mathrm{~cm}$ Int. Map. & $3.0^{*}$ & $\mathrm{M}$ & {$[92]$} \\
\hline ska1_lensing & SKA1 & Weak Lensing & $3.0^{*}$ & $\mathrm{M}$ & {$[92]$} \\
\hline ska1_pk & SKA1 & Galaxy Clust. & $3.0^{*}$ & $\mathrm{M}$ & {$[92]$} \\
\hline ska2_lensing & SKA2 & Weak Lensing & $3.0^{*}$ & $\mathrm{M}$ & {$[92]$} \\
\hline ska2_pk & SKA2 & Galaxy Clust. & $3.0^{*}$ & $\mathrm{M}$ & {$[92]$}
\end{tabular}

Table D.5: Forecast likelihoods. ${ }^{*}$ Euclid likelihoods will be updated and SKA likelihoods published when the relevant publication has been accepted for publication.

[1] B. Audren, J. Lesgourgues, K. Benabed, S. Prunet, Conservative Constraints on Early Cosmology: an illustration of the Monte Python cosmological parameter inference code, JCAP 1302 (2013) 001.

[2] A. Lewis, S. Bridle, Cosmological parameters from CMB and other data: A Monte Carlo approach, Phys. Rev. D66 (2002) 103511. arXiv: astro-ph/0205436 doi:10.1103/PhysRevD.66.103511

[3] A. Lewis, Efficient sampling of fast and slow cosmological parameters, Phys. Rev. D87 (10) (2013) 103529.

[4] J. Zuntz, M. Paterno, E. Jennings, D. Rudd, A. Manzotti, S. Dodelson, S. Bridle, S. Sehrish, J. Kowalkowski, CosmoSIS: Modular Cosmological Parameter Estimation, Astron. Comput. 12 (2015) 45-59. arXiv: 1409.3409 doi:10.1016/j.ascom.2015.05.005

[5] J. Lesgourgues, The Cosmic Linear Anisotropy Solving System (CLASS) I: Overview arXiv: 1104.2932

[6] D. Blas, J. Lesgourgues, T. Tram, The Cosmic Linear Anisotropy Solving System (CLASS) II: Approximation schemes, JCAP 1107 (2011) 034 .

[7] J. Lesgourgues, The Cosmic Linear Anisotropy Solving System (CLASS) III: Comparision with CAMB for LambdaCDMarXiv:1104. 2934

[8] J. Lesgourgues, T. Tram, The Cosmic Linear Anisotropy Solving System (CLASS) IV: efficient implementation of non-cold relics, JCAP 1109 (2011) 032.

[9] M. Zumalacárregui, E. Bellini, I. Sawicki, J. Lesgourgues, hi_class: Horndeski in the Cosmic Linear Anisotropy Solving SystemarXiv: 1605.06102

[10] G. W. Pettinari, C. Fidler, R. Crittenden, K. Koyama, D. Wands, The intrinsic bispectrum of the cosmic microwave background, J. Cosmology Astropart. Phys. 4 (2013) 3. arXiv:1302.0832 doi:10.1088/1475-7516/2013/04/003

[11] P. Stöcker, M. Krämer, J. Lesgourgues, V. Poulin, Exotic energy injection with ExoCLASS: Application to the Higgs portal model and evaporating black holes arXiv: 1801.01871

[12] B. Bolliet, B. Comis, E. Komatsu, J. F. Macías-Pérez, Dark Energy from the Thermal Sunyaev Zeldovich Power Spectrum arXiv:1712. 00788

[13] E. Di Dio, F. Montanari, J. Lesgourgues, R. Durrer, The CLASSgal code for Relativistic Cosmological Large Scale Structure, JCAP 1311 (2013) 044. arXiv:1307.1459 doi:10.1088/1475-7516/2013/11/044

[14] Y. Dirian, S. Foffa, M. Kunz, M. Maggiore, V. Pettorino, Non-local gravity and comparison with observational datasets. II. Updated results and Bayesian model comparison with $\Lambda$ CDM, JCAP 1605 (05) (2016) 068. arXiv:1602.03558 doi:10.1088/1475-7516/2016/05/068

[15] A. Lewis, A. Challinor, A. Lasenby, Efficient computation of CMB anisotropies in closed FRW models, Astrophys. J. 538 (2000) 473-476. arXiv:astro-ph/9911177 doi:10.1086/309179

[16] C. Howlett, A. Lewis, A. Hall, A. Challinor, CMB power spectrum parameter degeneracies in the era of precision cosmology, JCAP 1204 (2012) 027. arXiv:1201.3654 doi:10.1088/1475-7516/2012/04/027

[17] A. Refregier, L. Gamper, A. Amara, L. Heisenberg, PyCosmo: An Integrated Cosmological Boltzmann SolverarXiv: 1708.05177

[18] J. Dunkley, M. Bucher, P. G. Ferreira, K. Moodley, C. Skordis, Fast and reliable mcmc for cosmological parameter estimation, Mon. Not. Roy. Astron. Soc. 356 (2005) 925-936. arXiv: astro-ph/0405462 doi : 10.1111/j.1365-2966.2004.08464.x 
[19] A. Gelman, D. B. Rubin, Inference from Iterative Simulation Using Multiple Sequences, Statist. Sci. 7 (1992) $457-472$.

[20] H. Haario, E. Saksman, J. Tamminen, An adaptive metropolis algorithm Bernoulli 7 (2) (2001) 223-242. URL https://projecteuclid.org:443/euclid.bj/1080222083

[21] S. Henrot-Versillé, O. Perdereau, S. Plaszczynski, B. R. d'Orfeuil, M. Spinelli, M. Tristram, Agnostic cosmology in the CAMEL frameworkarXiv: 1607.02964

[22] B. Schroer, Forecasts on the sensitivity of future cosmological experiments using a new algorithm for bayesian parameter extraction, Bachelor thesis, TTK, RWTH Aachen University.

[23] D. Coe, Fisher Matrices and Confidence Ellipses: A Quick-Start Guide and Software arXiv:0906.4123

[24] L. Perotto, J. Lesgourgues, S. Hannestad, H. Tu, Y. Y. Y. Wong, Probing cosmological parameters with the CMB: Forecasts from full Monte Carlo simulations, JCAP 0610 (2006) 013. arXiv: astro-ph/0606227 doi:10.1088/1475-7516/2006/10/013

[25] M. Chevallier, D. Polarski, Accelerating universes with scaling dark matter, Int. J. Mod. Phys. D10 (2001) 213-224. arXiv: gr-qc/0009008. doi:10.1142/S0218271801000822

[26] E. V. Linder, Exploring the expansion history of the universe, Phys. Rev. Lett. 90 (2003) 091301. arXiv:astro-ph/0208512 doi: 10.1103/PhysRevLett.90.091301

[27] S. Hannestad, T. Schwetz, Cosmology and the neutrino mass ordering, JCAP 1611 (11) (2016) 035

[28] I. Esteban, M. C. Gonzalez-Garcia, M. Maltoni, I. Martinez-Soler, T. Schwetz, Updated fit to three neutrino mixing: exploring the acceleratorreactor complementarity, JHEP 01 (2017) 087

[29] S. Gariazzo, M. Archidiacono, P. F. de Salas, O. Mena, C. A. Ternes, M. Tórtola, Neutrino masses and their ordering: Global Data, Priors and Models, JCAP 1803 (03) (2018) 011. arXiv: 1801.04946 doi : 10.1088/1475-7516/2018/03/011

[30] E. Di Valentino, et al., Exploring Cosmic Origins with CORE: Cosmological ParametersarXiv: 1612.00021

[31] E. Giusarma, M. Gerbino, O. Mena, S. Vagnozzi, S. Ho, K. Freese, Improvement of cosmological neutrino mass bounds, Phys. Rev. D94 (8) (2016) 083522.

[32] W. Fang, W. Hu, A. Lewis, Crossing the Phantom Divide with Parameterized Post-Friedmann Dark Energy, Phys. Rev. D78 (2008) 087303. arXiv:0808.3125 doi:10.1103/PhysRevD.78.087303

[33] A. Upadhye, Neutrino mass and dark energy constraints from redshift-space distortions arXiv: 1707.09354

[34] P. A. R. Ade, et al., Planck 2015 results. XIII. Cosmological parameters, Astron. Astrophys. 594 (2016) A13. arXiv: 1502.01589 doi: 10.1051/0004-6361/201525830

[35] F. Feroz, M. P. Hobson, Multimodal nested sampling: an efficient and robust alternative to MCMC methods for astronomical data analysis, Mon. Not. Roy. Astron. Soc. 384 (2008) 449. arXiv:0704.3704 doi:10.1111/j.1365-2966.2007.12353.x

[36] F. Feroz, M. P. Hobson, M. Bridges, MultiNest: an efficient and robust Bayesian inference tool for cosmology and particle physics, Mon. Not. Roy. Astron. Soc. 398 (2009) 1601-1614. arXiv:0809.3437. doi:10.1111/j.1365-2966.2009.14548.x

[37] F. Feroz, M. P. Hobson, E. Cameron, A. N. Pettitt, Importance Nested Sampling and the MultiNest AlgorithmarXiv:1306.2144

[38] J. Buchner, A. Georgakakis, K. Nandra, L. Hsu, C. Rangel, M. Brightman, A. Merloni, M. Salvato, J. Donley, D. Kocevski, X-ray spectral modelling of the AGN obscuring region in the CDFS: Bayesian model selection and catalogue, Astron. Astrophys. 564 (2014) A125. arXiv: 1402.0004 doi:10.1051/0004-6361/201322971

[39] J. Goodman, J. Weare, Ensemble samplers with affine invariance, Communications in Applied Mathematics and Computational Science, Vol. 5, No. 1, p. 65-80, 20105 (2010) 65-80. doi:10.2140/camcos.2010.5.65

[40] D. Foreman-Mackey, D. W. Hogg, D. Lang, J. Goodman, emcee: The MCMC Hammer, Publ. Astron. Soc. Pac. 125 (2013) $306-312$. arXiv:1202.3665 doi:10.1086/670067

[41] J. Akeret, S. Seehars, A. Amara, A. Refregier, A. Csillaghy, CosmoHammer: Cosmological parameter estimation with the MCMC HammerarXiv: 1212.1721

[42] C.-L. Kuo, et al., Improved Measurements of the CMB Power Spectrum with ACBAR, Astrophys. J. 664 (2007) 687-701. arXiv : astro-ph/ 0611198 doi:10.1086/518401

[43] F. Beutler, C. Blake, M. Colless, D. H. Jones, L. Staveley-Smith, L. Campbell, Q. Parker, W. Saunders, F. Watson, The 6dF Galaxy Survey: Baryon Acoustic Oscillations and the Local Hubble Constant, Mon. Not. Roy. Astron. Soc. 416 (2011) 3017-3032. arXiv:1106.3366. doi:10.1111/j.1365-2966.2011.19250.x

[44] L. Anderson, et al., The clustering of galaxies in the SDSS-III Baryon Oscillation Spectroscopic Survey: Baryon Acoustic Oscillations in the Data Release 9 Spectroscopic Galaxy Sample, Mon. Not. Roy. Astron. Soc. 427 (4) (2013) 3435-3467. arXiv:1203.6594. doi: 10.1111/j.1365-2966.2012.22066.x

[45] A. J. Ross, L. Samushia, C. Howlett, W. J. Percival, A. Burden, M. Manera, The clustering of the SDSS DR7 main Galaxy sample - I. A 4 per cent distance measure at $z=0.15$, Mon. Not. Roy. Astron. Soc. 449 (1) (2015) 835-847. arXiv:1409.3242 doi:10.1093/mnras/ stv154

[46] B. Audren, Separate Constraints on Early and Late Cosmology, Mon. Not. Roy. Astron. Soc. 444 (1) (2014) 827-832. arXiv: 1312.5696. doi:10.1093/mnras/stu1457

[47] E. Sanchez, A. Carnero, J. Garcia-Bellido, E. Gaztanaga, F. de Simoni, M. Crocce, A. Cabre, P. Fosalba, D. Alonso, Tracing The Sound Horizon Scale With Photometric Redshift Surveys, Mon. Not. Roy. Astron. Soc. 411 (2011) 277-288. arXiv:1006.3226 doi:10.1111/ j.1365-2966.2010.17679.x

[48] G. C. Carvalho, A. Bernui, M. Benetti, J. C. Carvalho, J. S. Alcaniz, Baryon Acoustic Oscillations from the SDSS DR10 galaxies angular correlation function, Phys. Rev. D93 (2) (2016) 023530. arXiv:1507.08972 doi:10.1103/PhysRevD.93.023530

[49] J. S. Alcaniz, G. C. Carvalho, A. Bernui, J. C. Carvalho, M. Benetti, Measuring baryon acoustic oscillations with angular two-point correlation function, Fundam. Theor. Phys. 187 (2017) 11-19. arXiv:1611.08458 doi:10.1007/978-3-319-51700-1_2

[50] E. de Carvalho, A. Bernui, G. C. Carvalho, C. P. Novaes, Angular Baryon Acoustic Oscillation measure at $\mathrm{z}=2.225$ from the SDSS quasar survey arXiv:1709.00113

[51] G. C. Carvalho, A. Bernui, M. Benetti, J. C. Carvalho, J. S. Alcaniz, Measuring the transverse baryonic acoustic scale from the SDSS DR11 galaxiesarXiv:1709.00271 
[52] L. Anderson, et al., The clustering of galaxies in the SDSS-III Baryon Oscillation Spectroscopic Survey: baryon acoustic oscillations in the Data Releases 10 and 11 Galaxy samples, Mon. Not. Roy. Astron. Soc. 441 (1) (2014) 24-62. arXiv:1312.4877. doi:10.1093/mnras/ stu523

[53] S. Alam, et al., The clustering of galaxies in the completed SDSS-III Baryon Oscillation Spectroscopic Survey: cosmological analysis of the DR12 galaxy sample, Mon. Not. Roy. Astron. Soc. 470 (3) (2017) 2617-2652. arXiv: 1607.03155 doi:10.1093/mnras/stx721

[54] M. A. Buen-Abad, M. Schmaltz, J. Lesgourgues, T. Brinckmann, Interacting Dark Sector and Precision Cosmology, JCAP 1801 (01) (2018) 008. arXiv: 1708.09406 doi:10.1088/1475-7516/2018/01/008

[55] H. C. Chiang, et al., Measurement of CMB Polarization Power Spectra from Two Years of BICEP Data, Astrophys. J. 711 (2010) $1123-1140$. arXiv:0906.1181 doi:10.1088/0004-637X/711/2/1123

[56] P. A. R. Ade, et al., Detection of B-Mode Polarization at Degree Angular Scales by BICEP2, Phys. Rev. Lett. 112 (24) (2014) 241101. arXiv:1403.3985 doi:10.1103/PhysRevLett.112.241101

[57] P. A. R. Ade, et al., Improved Constraints on Cosmology and Foregrounds from BICEP2 and Keck Array Cosmic Microwave Background Data with Inclusion of 95 GHz Band, Phys. Rev. Lett. 116 (2016) 031302. arXiv:1510.09217 doi:10.1103/PhysRevLett.116. 031302

[58] W. C. Jones, et al., A Measurement of the angular power spectrum of the CMB temperature anisotropy from the 2003 flight of BOOMERANG, Astrophys. J. 647 (2006) 823-832. arXiv:astro-ph/0507494 doi:10.1086/505559

[59] J. L. Sievers, et al., Implications of the cosmic background imager polarization data, Astrophys. J. 660 (2007) 976-987. arXiv:astro-ph/ 0509203 doi:10.1086/510504

[60] C. Heymans, et al., CFHTLenS tomographic weak lensing cosmological parameter constraints: Mitigating the impact of intrinsic galaxy alignments, Mon. Not. Roy. Astron. Soc. 432 (2013) 2433. arXiv:1303.1808 doi:10.1093/mnras/stt601

[61] D. Larson, et al., Seven-Year Wilkinson Microwave Anisotropy Probe (WMAP) Observations: Power Spectra and WMAP-Derived Parameters, Astrophys. J. Suppl. 192 (2011) 16. arXiv: 1001.4635 doi:10.1088/0067-0049/192/2/16

[62] M. Moresco, L. Pozzetti, A. Cimatti, R. Jimenez, C. Maraston, L. Verde, D. Thomas, A. Citro, R. Tojeiro, D. Wilkinson, A 6\% measurement of the Hubble parameter at $z \sim 0.45$ : direct evidence of the epoch of cosmic re-acceleration, JCAP 1605 (05) (2016) 014. arXiv: 1601.01701. doi:10.1088/1475-7516/2016/05/014

[63] M. Moresco, et al., Improved constraints on the expansion rate of the Universe up to $\mathrm{z} 1.1$ from the spectroscopic evolution of cosmic chronometers, JCAP 1208 (2012) 006. arXiv: 1201.3609 doi:10.1088/1475-7516/2012/08/006

[64] J. Simon, L. Verde, R. Jimenez, Constraints on the redshift dependence of the dark energy potential, Phys. Rev. D71 (2005) 123001. arXiv: astro-ph/0412269 doi:10.1103/PhysRevD.71.123001

[65] D. Stern, R. Jimenez, L. Verde, M. Kamionkowski, S. A. Stanford, Cosmic Chronometers: Constraining the Equation of State of Dark Energy. I: H(z) Measurements, JCAP 1002 (2010) 008. arXiv: 0907.3149 doi:10.1088/1475-7516/2010/02/008

[66] X.-H. Fan, C. L. Carilli, B. G. Keating, Observational constraints on cosmic reionization, Ann. Rev. Astron. Astrophys. 44 (2006) $415-462$. arXiv:astro-ph/0602375 doi:10.1146/annurev.astro.44.051905.092514

[67] A. G. Riess, et al., A 2.4\% Determination of the Local Value of the Hubble Constant, Astrophys. J. 826 (1) (2016) 56. arXiv: 1604.01424. doi: $10.3847 / 0004-637 \mathrm{X} / 826 / 1 / 56$

[68] J. Schaye, T. Theuns, M. Rauch, G. Efstathiou, W. L. W. Sargent, The Thermal history of the intergalactic medium, Mon. Not. Roy. Astron. Soc. 318 (2000) 817. arXiv:astro-ph/9912432 doi:10.1046/j.1365-8711.2000.03815.x

[69] B. Stölzner, A. Cuoco, J. Lesgourgues, M. Bilicki, An Updated Tomographic Analysis of the Integrated Sachs-Wolfe Effect and implications for Dark Energy arXiv: 1710.03238

[70] M. Betoule, et al., Improved cosmological constraints from a joint analysis of the SDSS-II and SNLS supernova samples, Astron. Astrophys. 568 (2014) A22. arXiv: 1401.4064 doi:10.1051/0004-6361/201423413

[71] F. Köhlinger, et al., KiDS-450: The tomographic weak lensing power spectrum and constraints on cosmological parameters, Mon. Not. Roy. Astron. Soc. 471 (4) (2017) 4412-4435. arXiv: 1706.02892 doi:10.1093/mnras/stx1820

[72] P. A. R. Ade, et al., Planck 2013 results. XV. CMB power spectra and likelihood, Astron. Astrophys. 571 (2014) A15. arXiv:1303.5075. doi:10.1051/0004-6361/201321573

[73] J. Dunkley, E. Calabrese, J. Sievers, G. E. Addison, N. Battaglia, E. S. Battistelli, J. R. Bond, S. Das, M. J. Devlin, R. Dünner, J. W. Fowler, M. Gralla, A. Hajian, M. Halpern, M. Hasselfield, A. D. Hincks, R. Hlozek, J. P. Hughes, K. D. Irwin, A. Kosowsky, T. Louis, T. A. Marriage, D. Marsden, F. Menanteau, K. Moodley, M. Niemack, M. R. Nolta, L. A. Page, B. Partridge, N. Sehgal, D. N. Spergel, S. T. Staggs, E. R. Switzer, H. Trac, E. Wollack, The Atacama Cosmology Telescope: likelihood for small-scale CMB data, JCAP 7 (2013) 025. arXiv:1301.0776 doi:10.1088/1475-7516/2013/07/025

[74] R. Keisler, C. L. Reichardt, K. A. Aird, B. A. Benson, L. E. Bleem, J. E. Carlstrom, C. L. Chang, H. M. Cho, T. M. Crawford, A. T. Crites, T. de Haan, M. A. Dobbs, J. Dudley, E. M. George, N. W. Halverson, G. P. Holder, W. L. Holzapfel, S. Hoover, Z. Hou, J. D. Hrubes, M. Joy, L. Knox, A. T. Lee, E. M. Leitch, M. Lueker, D. Luong-Van, J. J. McMahon, J. Mehl, S. S. Meyer, M. Millea, J. J. Mohr, T. E. Montroy, T. Natoli, S. Padin, T. Plagge, C. Pryke, J. E. Ruhl, K. K. Schaffer, L. Shaw, E. Shirokoff, H. G. Spieler, Z. Staniszewski, A. A. Stark, K. Story, A. van Engelen, K. Vanderlinde, J. D. Vieira, R. Williamson, O. Zahn, A Measurement of the Damping Tail of the Cosmic Microwave Background Power Spectrum with the South Pole Telescope, Astrophys. J. 743 (2011) 28. arXiv:1105.3182. doi: $10.1088 / 0004-637 \mathrm{X} / 743 / 1 / 28$

[75] N. Aghanim, et al., Planck 2015 results. XI. CMB power spectra, likelihoods, and robustness of parameters, Astron. Astrophys. 594 (2016) A11. arXiv: 1507.02704 doi:10.1051/0004-6361/201526926

[76] P. A. R. Ade, et al., Planck 2015 results. XV. Gravitational lensing, Astron. Astrophys. 594 (2016) A15. arXiv:1502.01591 doi:10. 1051/0004-6361/201525941

[77] P. A. R. Ade, et al., Planck 2015 results. XXIV. Cosmology from Sunyaev-Zeldovich cluster counts, Astron. Astrophys. 594 (2016) A24. arXiv: 1502.01597 doi:10.1051/0004-6361/201525833

[78] P. A. R. Ade, et al., A Measurement of the Cosmic Microwave Background B-Mode Polarization Power Spectrum at Sub-Degree Scales with POLARBEAR, Astrophys. J. 794 (2) (2014) 171. arXiv:1403.2369 doi:10.1088/0004-637X/794/2/171 
[79] M. L. Brown, et al., Improved measurements of the temperature and polarization of the CMB from QUaD, Astrophys. J. 705 (2009) $978-999$. arXiv:0906.1003 doi:10.1088/0004-637X/705/1/978

[80] M. Tegmark, et al., Cosmological Constraints from the SDSS Luminous Red Galaxies, Phys. Rev. D74 (2006) 123507. arXiv:astro-ph/ 0608632 doi:10.1103/PhysRevD.74.123507

[81] B. A. Reid, et al., Cosmological Constraints from the Clustering of the Sloan Digital Sky Survey DR7 Luminous Red Galaxies, Mon. Not. Roy. Astron. Soc. 404 (2010) 60-85. arXiv:0907.1659 doi:10.1111/j.1365-2966.2010.16276.x

[82] N. Aghanim, et al., Planck intermediate results. XLVI. Reduction of large-scale systematic effects in HFI polarization maps and estimation of the reionization optical depth, Astron. Astrophys. 596 (2016) A107. arXiv:1605.02985 doi:10.1051/0004-6361/201628890

[83] R. Adam, et al., Planck intermediate results. XLVII. Planck constraints on reionization history, Astron. Astrophys. 596 (2016) A108. arXiv: 1605.03507 doi:10.1051/0004-6361/201628897

[84] R. Amanullah, et al., Spectra and Light Curves of Six Type Ia Supernovae at $0.511 ; \mathrm{z} ; 1.12$ and the Union 2 Compilation, Astrophys. J. 716 (2010) 712-738. arXiv:1004.1711 doi:10.1088/0004-637X/716/1/712

[85] K. K. Schaffer, et al., The First Public Release of South Pole Telescope Data: Maps of a 95-square-degree Field from 2008 Observations, Astrophys. J. 743 (2011) 90. arXiv:1111.7245 doi:10.1088/0004-637X/743/1/90

[86] S. H. Suyu, et al., Two accurate time-delay distances from strong lensing: Implications for cosmology, Astrophys. J. 766 (2013) 70. arXiv: 1208.6010 doi:10.1088/0004-637X/766/2/70

[87] D. Parkinson, et al., The WiggleZ Dark Energy Survey: Final data release and cosmological results, Phys. Rev. D86 (2012) 103518. arXiv: 1210.2130 doi:10.1103/PhysRevD.86.103518

[88] E. A. Kazin, et al., The WiggleZ Dark Energy Survey: improved distance measurements to $\mathrm{z}=1$ with reconstruction of the baryonic acoustic feature, Mon. Not. Roy. Astron. Soc. 441 (4) (2014) 3524-3542. arXiv:1401.0358 doi:10.1093/mnras/stu778

[89] C. L. Bennett, et al., Nine-Year Wilkinson Microwave Anisotropy Probe (WMAP) Observations: Final Maps and Results, Astrophys. J. Suppl. 208 (2013) 20. arXiv:1212.5225 doi:10.1088/0067-0049/208/2/20

[90] F. Finelli, et al., Exploring Cosmic Origins with CORE: Inflation arXiv: 1612.08270

[91] B. Audren, J. Lesgourgues, S. Bird, M. G. Haehnelt, M. Viel, Neutrino masses and cosmological parameters from a Euclid-like survey: Markov Chain Monte Carlo forecasts including theoretical errors, JCAP 1301 (2013) 026. arXiv:1210.2194 doi:10.1088/1475-7516/ 2013/01/026

[92] T. Sprenger, M. Archidiacono, T. Brinckmann, S. Clesse, J. Lesgourgues, Cosmology in the era of Euclid and the Square Kilometre ArrayarXiv:1801.08331

[93] A. Font-Ribera, P. McDonald, N. Mostek, B. A. Reid, H.-J. Seo, A. Slosar, DESI and other dark energy experiments in the era of neutrino mass measurements, JCAP 1405 (2014) 023. arXiv: 1308.4164 doi:10.1088/1475-7516/2014/05/023

[94] M. Archidiacono, T. Brinckmann, J. Lesgourgues, V. Poulin, Physical effects involved in the measurements of neutrino masses with future cosmological data, JCAP 1702 (02) (2017) 052. arXiv:1610.09852 doi:10.1088/1475-7516/2017/02/052

[95] J. Tauber, M. Bersanelli, J. M. Lamarre, G. Efstathiou, C. Lawrence, F. Bouchet, E. Martinez-Gonzalez, S. Matarrese, D. Scott, M. White, et al., The Scientific programme of PlanckarXiv:astro-ph/0604069

[96] A. Suzuki, et al., The LiteBIRD Satellite Mission - Sub-Kelvin Instrument in: 17th International Workshop on Low Temperature Detectors (LTD 17) Kurume City, Japan, July 17-21, 2017, 2018. arXiv:1801.06987 URL https://inspirehep.net/record/1649560/files/arXiv:1801.06987.pdf 